\title{
Optical Spectroscopy of Type la Supernovae
}

\section{Citation}

Matheson, T., R. P. Kirshner, P. Challis, S. Jha, P. M. Garnavich, P. Berlind, M. L. Calkins, et al. 2008. "Optical Spectroscopy of Type la Supernovae" The Astronomical Journal 135 (4) (March 13): 1598-1615. doi:10.1088/0004-6256/135/4/1598.

\section{Published Version}

doi:10.1088/0004-6256/135/4/1598

\section{Permanent link}

http://nrs.harvard.edu/urn-3:HUL.InstRepos:29921877

\section{Terms of Use}

This article was downloaded from Harvard University's DASH repository, and is made available under the terms and conditions applicable to Other Posted Material, as set forth at http:// nrs.harvard.edu/urn-3:HUL.InstRepos:dash.current.terms-of-use\#LAA

\section{Share Your Story}

The Harvard community has made this article openly available.

Please share how this access benefits you. Submit a story.

Accessibility 


\title{
OPTICAL SPECTROSCOPY OF TYPE Ia SUPERNOVAE*
}

\author{
T. Matheson ${ }^{1}$, R. P. Kirshner ${ }^{2}$, P. Challis ${ }^{2}$, S. Jha ${ }^{3}$, P. M. Garnavich ${ }^{4}$, P. Berlind ${ }^{5}$, M. L. Calkins ${ }^{5}$, S. Blondin ${ }^{2}$, \\ Z. Balog ${ }^{6,13}$, A. E. BragG ${ }^{7}$, N. Calddwell ${ }^{2}$, K. Dendy Concannon ${ }^{8}$, E. E. Falco ${ }^{5}$, G. J. M. Graves ${ }^{9}$, J. P. Huchra ${ }^{2}$,

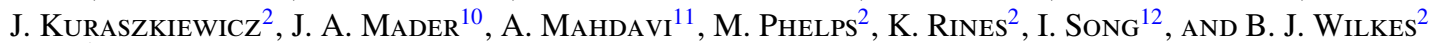 \\ ${ }^{1}$ National Optical Astronomy Observatory, 950 N. Cherry Avenue, Tucson, AZ 85719-4933, USA; matheson@ noao.edu \\ ${ }^{2}$ Harvard-Smithsonian Center for Astrophysics, 60 Garden Street, Cambridge, MA 02138, USA; kirshner@cfa.harvard.edu, pchallis@cfa.harvard.edu, \\ sblondin@cfa.harvard.edu,ncaldwell@cfa.harvard.edu,jhuchra@cfa.harvard.edu,jkuraszkiewicz@cfa.harvard.edu,mphelps@cfa.harvard.edu, and \\ krines@cfa.harvard.edu \\ ${ }^{3}$ Kavli Institute for Particle Astrophysics and Cosmology, P.O. Box 20450, Stanford, CA 94309, USA; saurabh@ slac.stanford.edu \\ ${ }^{4}$ Department of Physics, University of Notre Dame, 225 Nieuwland Science Hall, Notre Dame, IN 46556-5670, USA; pgarnavi@nd.edu \\ ${ }^{5}$ F. L. Whipple Observatory, 670 Mt. Hopkins Road, P.O. Box 97, Amado, AZ 85645, USA; pberlind@cfa.harvard.edu, mcalkins@cfa.harvard.edu, and \\ efalco@cfa.harvard.edu \\ ${ }^{6}$ Steward Observatory, University of Arizona, 933. N. Cherry Avenue Tucson, AZ 85721, USA; zbalog@as.arizona.edu \\ ${ }^{7}$ Department of Physics and Astronomy, Bowling Green State University, Bowling Green, OH 43403, USA; aebragg@ bgnet.bgsu.edu \\ ${ }^{8}$ Department of Chemistry and Physics, King's College, 133 North River Street, Wilkes-Barre, PA 18711, USA; kdconcan@kings.edu \\ ${ }^{9}$ UCO/Lick Observatory, University of California, Santa Cruz, CA 95064, USA; graves@ ucolick.org \\ ${ }^{10}$ W. M. Keck Observatory, 65-1120 Mamalahoa Highway, Kamuela, HI 96743, USA; jmader@keck.hawaii.edu \\ ${ }^{11}$ Department of Physics and Astronomy, University of Victoria, Victoria, BC V8W 3P6, Canada; amahdavi@uvic.ca \\ 12 Spitzer Science Center, IPAC/Caltech, Pasadena, CA 91125, USA; song@ipac.caltech.edu \\ Received 2007 August 28; accepted 2007 December 26; published 2008 March 13
}

\begin{abstract}
We present 432 low-dispersion optical spectra of 32 Type Ia supernovae (SNe Ia) that also have well-calibrated light curves. The coverage ranges from 6 epochs to 36 epochs of spectroscopy. Most of the data were obtained with the $1.5 \mathrm{~m}$ Tillinghast telescope at the F. L. Whipple Observatory with typical wavelength coverage of 3700-7400 and a resolution of $\sim 7 \AA$. The earliest spectra are 13 days before $B$-band maximum; two-thirds of the $\mathrm{SNe}$ were observed before maximum brightness. Coverage for some SNe continues almost to the nebular phase. The consistency of the method of observation and the technique of reduction makes this an ideal data set for studying the spectroscopic diversity of SNe Ia.
\end{abstract}

Key words: supernovae: general - surveys

Online-only material: color figure, machine-readable table

\section{INTRODUCTION}

Type Ia supernovae (SNe Ia) have long been intriguing objects for astronomers. As individual objects, they present complex problems about the nature of their progenitors (e.g., Howell et al. 2001; Branch 2001; Nomoto et al. 2003; Stritzinger et al. 2006, and references therein), the physics of the explosion mechanism (e.g., Woosley \& Weaver 1986; Hillebrandt \& Niemeyer 2000; Gamezo et al. 2004, and references therein), and the factors that produce the observed range of diversity (e.g., Hatano et al. 2000; Li et al. 2001b; Benetti et al. 2005). In recent years, a great deal of attention has been focused on the fact that absolute magnitudes of SNe Ia can be deduced from the shape of their light curves (e.g., Phillips 1993; Hamuy et al. 1996a; Riess et al. 1996; Perlmutter et al. 1997; Jha et al. 2007). Once this calibration has been applied, $\mathrm{SNe}$ Ia are the best extragalactic distance measuring tools. Combined with their large intrinsic brightness, this makes SNe Ia extremely valuable as cosmological distance indicators. Using $\mathrm{SNe}$ Ia as cosmological lighthouses led to a recent revolution in cosmology, with the discovery that the universe was accelerating (e.g., Riess et al. 1998, 2001, 2004, 2007; Perlmutter et al.

\footnotetext{
* Based in part on observations obtained at the F. L. Whipple Observatory, which is operated by the Smithsonian Astrophysical Observatory, and the MMT Observatory, a joint facility of the Smithsonian Institution and the University of Arizona.

${ }^{13}$ On leave from the Department of Optics and Quantum Electronics,

University of Szeged, Dóm tér 9, H-6720 Szeged, Hungary.
}

1999; Knop et al. 2003; Tonry et al. 2003; Astier et al. 2006; Wood-Vasey et al. 2007), contrary to all expectations. The nature of the dark energy that is producing the acceleration is one of the great unanswered questions of current physics.

For questions associated with understanding individual $\mathrm{SNe} \mathrm{Ia}$, as well as those related to their use as high-redshift distance indicators, the quality of the answers will be based upon the underlying data that are used to make inferences. There have been much data published about SNe Ia, but most have been from detailed studies of individual objects, with early pioneering work on SN 1972E (Kirshner et al. 1973; Kirshner \& Oke 1975) up to more recent studies (e.g., Kirshner et al. 1993; Stritzinger et al. 2002; Krisciunas et al. 2003; Benetti et al. 2004; Pignata et al. 2004; Kotak et al. 2005). The extreme examples of SNe Ia, such as the overluminous SN 1991T (Filippenko et al. 1992a; Phillips et al. 1992) and the underluminous SN 1991bg (Filippenko et al. 1992b; Leibundgut et al. 1993), have also been well studied. The drawbacks of earlier samples of SN spectra were the heterogeneous nature of the data and the relatively small size of the sample. Examples of the data can be seen at the University of Oklahoma's supernova spectra database (SUSPECT, http://bruford.nhn.ou.edu/ suspect/index1.html) or the Web site of the European Research Training Network on the Physics of Type Ia Supernova Explosions (http://www.mpa-garching.mpg.de/ rtn/). The spectra were often obtained at a variety of sites and reduced in different ways. For photometry alone, there are several large, homogeneous data sets that have been published (Hamuy et al. 1996b; Riess 
et al. 1999; Jha et al. 2006), consisting of light curves of a wide variety of SNe Ia. There have been no large, homogeneous data sets of spectra of SNe Ia. Such a sample will have a wide variety of applications, from testing explosion models to understanding systematic errors that plague the use of SNe Ia as cosmological distance indicators. Although most attention is focused on the photospheric-phase spectra, the nebular-phase spectra can reveal much about SNe Ia. Nebular lines in time-series spectra of SNe Ia show direct evidence for the changing ratio of cobalt and iron lines, implying that they are powered by radioactive iron-peak elements (Kuchner et al. 1994). In addition, Mazzali et al. (1998) showed that the width of nebular lines was related to the luminosity of the SN.

Two of the large light-curve data sets mentioned above (Riess et al. 1999; Jha et al. 2006) are the result of a program begun in 1993 by the SN group at the Harvard-Smithsonian Center for Astrophysics (CfA) to monitor SNe (of all types) photometrically and spectroscopically with the telescopes at the F. L. Whipple Observatory (FLWO) on Mt. Hopkins, Arizona. Through the use of queue scheduling for spectroscopic observations and a cooperative strategy of a small allocation of photometric time per night, we have been able to obtain data with a frequent enough cadence to acquire good data sets on many objects. The early spectroscopic coverage was mainly for classification, but, starting in 1997, we began to follow objects in earnest. Classification is still a major part of the program; between 2000 September and 2003 September, we classified $39 \%$ of the low-redshift $\mathrm{SNe}$ accessible from the Northern Hemisphere.

In this paper, we present the first release of some of the spectroscopic data obtained at Mt. Hopkins by the CfA SN group between 1997 and early 2001. The decision to follow a specific SN Ia with extensive spectroscopic coverage was based upon apparent brightness, availability of telescope time, and the relative phase of the SN at our first spectrum. For the purposes of the sample presented here, we only include $\mathrm{SNe}$ Ia for which we have a reasonable number of spectra $(>6)$. The final criterion for selecting objects from the CfA SN database was whether or not there was a calibrated light curve. With a light curve, the epoch of maximum is established. This also gives us the potential to correlate the light-curve shape with spectroscopic properties, which is an important goal for this program. Most of the photometry for the spectroscopic sample in this paper is presented by Jha et al. (2006). A few of these SNe Ia were published as single objects: (SN 1998aq, Riess et al. 2005), (SN 1998bu, Jha et al. 1999c), (SN 1999by, Garnavich et al. 2004). Some SNe from 2000 await final photometric calibration, and so are not included here. In total, there were $32 \mathrm{SNe}$ Ia that fit all the criteria, with 432 individual spectra. A histogram of the number of epochs of spectroscopy for the 14 days before and after maximum brightness is shown in Figure 1. Many of the objects were observed well before maximum brightness. Figure 2 shows the histogram of the epoch of the SN at the first spectrum. In addition, the $\mathrm{SNe}$ span the known range of $\Delta m_{15}(B)$ (Figure 3). The $\mathrm{SNe}$ selected, along with some properties of the host galaxies, are listed in Table 1. Virtually all the spectra are from the same instrument, and they have all reduced in the same manner. In a companion paper (T. Matheson et al. 2008, in preparation), we will present a preliminary analysis of the spectroscopic characteristics of this data set. In that paper, we will relate spectroscopic properties to the light-curve shapes of the SNe, specifically how the strength of the silicon features correlates with decline rate. In addition, we will show

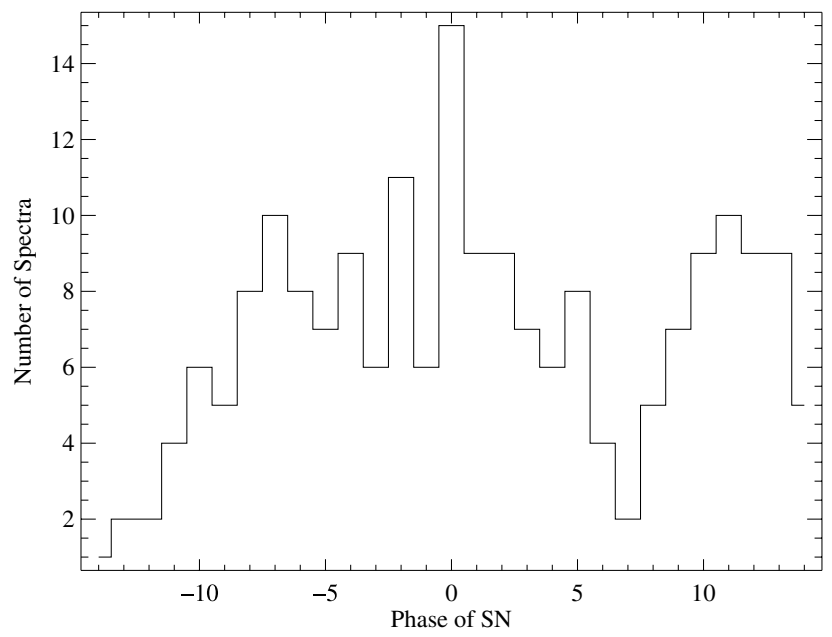

Figure 1. Histogram of the number of individual SN spectra at each epoch within 14 days of maximum brightness.

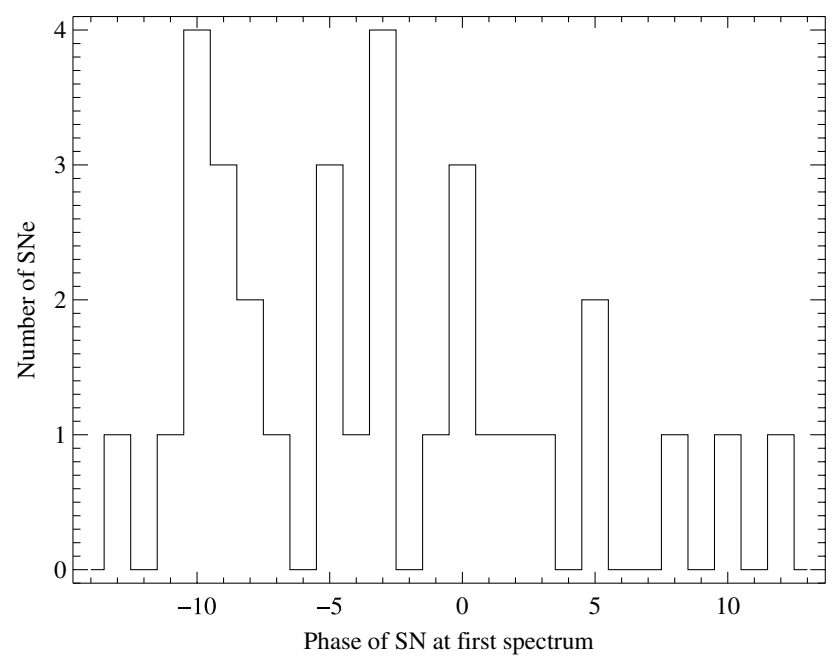

Figure 2. Histogram of the number of $\mathrm{SNe}$ at the epoch of the first spectrum.

the degree of variation within the SNe Ia and how this changes with decline rate.

All the spectra presented in this paper will be made publicly available through the CfA Supernova Archive (http://www.cfa.harvard.edu/supernova/SNarchive.html). This archive contains all published data from the CfA SN group, both photometric and spectroscopic.

\section{OBSERVATIONS}

The majority of the spectra presented in this paper were obtained with the $1.5 \mathrm{~m}$ Tillinghast telescope at FLWO using the FAST spectrograph (Fabricant et al. 1998). Spectroscopic observations with the Tillinghast (both low-dispersion with FAST such as are discussed here and high-dispersion echelle spectra) are made in a queue-scheduled mode. Two professional observers (P. Berlind \& M. L. Calkins) were the primary observers during the period when these spectra were taken. In addition, portions of the telescope schedule are staffed by other CfA personnel. The CfA SN group would request two or three observations per night when FAST was scheduled, subject to constraints at the telescope (e.g., weather, instrument 
Table 1

SN Ia and Host Basic Data

\begin{tabular}{|c|c|c|c|c|}
\hline SN & $\begin{array}{l}\text { Host } \\
\text { galaxy }\end{array}$ & $\begin{array}{c}c z_{\text {helio }}{ }^{\mathrm{a}} \\
\left(\mathrm{km} \mathrm{s}^{-1}\right)\end{array}$ & Host morph. ${ }^{b}$ & $\begin{array}{c}E(B-V)_{\text {Galactic }}{ }^{\mathrm{c}} \\
(\mathrm{mag})\end{array}$ \\
\hline SN 1997do & UGC 3845 & 3034 & Sbc & 0.063 \\
\hline SN 1997dt & NGC 7448 & 2194 & $\mathrm{Sbc}$ & 0.057 \\
\hline SN 1998V & NGC 6627 & 5268 & $\mathrm{Sb}$ & 0.196 \\
\hline SN 1998ab & NGC 4704 & 8134 & $\mathrm{Sc}$ & 0.017 \\
\hline SN 1998aq & NGC 3982 & 1184 & $\mathrm{Sb}$ & 0.014 \\
\hline SN 1998bp & NGC 6495 & 3127 & $\mathrm{E}$ & 0.076 \\
\hline SN 1998bu & NGC 3368 & 897 & $\mathrm{Sab}$ & 0.025 \\
\hline SN 1998de & NGC 252 & 4990 & S0 & 0.057 \\
\hline SN 1998dh & NGC 7541 & 2678 & $\mathrm{Sbc}$ & 0.068 \\
\hline SN 1998dk & UGC 139 & 3963 & $\mathrm{Sc}$ & 0.044 \\
\hline SN 1998dm & MCG-01-4-44 & 1968 & $\mathrm{Sc}$ & 0.044 \\
\hline SN 1998ec & UGC 3576 & 5966 & $\mathrm{Sb}$ & 0.085 \\
\hline SN 1998eg & UGC 12133 & 7423 & $\mathrm{Sc}$ & 0.123 \\
\hline SN 1998es & NGC 632 & 3168 & So & 0.032 \\
\hline SN 1999X & CGCG 180-22 & 7503 & $\cdots$ & 0.032 \\
\hline SN 1999aa & NGC 2595 & 4330 & Sc & 0.040 \\
\hline SN 1999ac & NGC 6063 & 2848 & Scd & 0.046 \\
\hline SN 1999by & NGC 2841 & 638 & $\mathrm{Sb}$ & 0.016 \\
\hline SN 1999cc & NGC 6038 & 9392 & $\mathrm{Sc}$ & 0.023 \\
\hline SN 1999cl & NGC 4501 (M88) & 2281 & $\mathrm{Sb}$ & 0.038 \\
\hline SN 1999dq & NGC 976 & 4295 & $\mathrm{Sc}$ & 0.110 \\
\hline SN 1999ej & NGC 495 & 4114 & $\mathrm{~S} 0 / \mathrm{Sa}$ & 0.071 \\
\hline SN 1999gd & NGC 2623 & 5535 & $\mathrm{Sa}$ & 0.041 \\
\hline SN 1999gh & NGC 2986 & 2302 & $\mathrm{E}$ & 0.058 \\
\hline SN 1999gp & UGC 1993 & 8018 & $\mathrm{Sb}$ & 0.056 \\
\hline SN 2000B & NGC 2320 & 5901 & $\mathrm{E}$ & 0.068 \\
\hline SN 2000cf & MCG+11-19-25 & 10920 & $\mathrm{Sbc}$ & 0.032 \\
\hline SN 2000cn & UGC 11064 & 7043 & Scd & 0.057 \\
\hline SN 2000cx & NGC 524 & 2379 & So & 0.082 \\
\hline SN 2000dk & NGC 382 & 5228 & E & 0.070 \\
\hline SN 2000fa & UGC 3770 & 6378 & $\mathrm{Sd} / \mathrm{Irr}$ & 0.069 \\
\hline SN 2001V & NGC 3987 & 4502 & $\mathrm{Sb}$ & 0.020 \\
\hline
\end{tabular}

Notes.

${ }^{a}$ Heliocentric redshifts listed are from the Updated Zwicky Catalog (Falco et al. 1999) if possible, and from the NASA/IPAC Extragalactic Database (NED) if not. For the host of SN 2000cf (MCG+11-19-25), we derived the velocity of $10,920 \mathrm{~km} \mathrm{~s}^{-1}$ from our own spectra of the host galaxy.

${ }^{\mathrm{b}}$ Host galaxy morphology is taken from NED, if possible, and from the LEDA database if not

c The Galactic reddening toward each SN is derived from the dust maps of Schlegel et al. (1998).

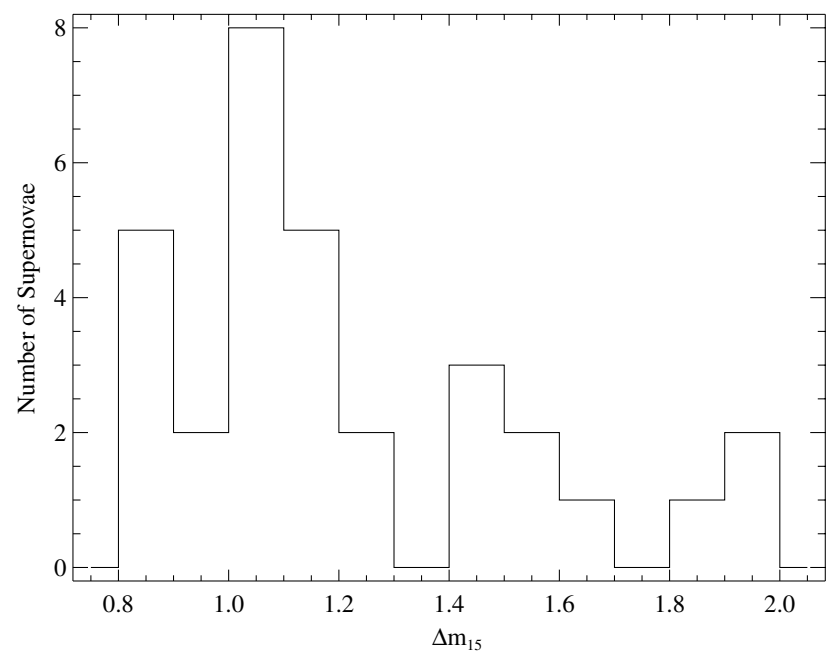

Figure 3. Histogram of the number of $\mathrm{SNe}$ versus $\Delta m_{15}(B)$ (from Jha et al. 2006). problems, conflicts with other programs). Observational details of the spectra are listed in Table 2.

The FAST spectrograph uses a $2688 \times 512$ Loral chargedcoupled device (CCD) with a spatial scale of 1.'1 per pixel in the binning mode used for these observations. The grating used yields a resolution of $\sim 7 \AA$. The usual setup for observations covered the usable wavelength range of $\sim 3700$ to $\sim 7500 \AA$. Slight variations in the wavelength range were sometimes introduced during instrument changes. Other programs in the FAST queue might occasionally require different spectrograph settings, resulting in different wavelength ranges. In addition, for bright and unusual SNe, we would request multiple observations with different grating tilts to observe a broader wavelength range. The typical slit width was $3^{\prime \prime}$. For most of the observations obtained in 1997 and 1998, the slit was oriented with a position angle of $90^{\circ}$. Starting in late 1998 , the slit was typically repositioned to the parallactic angle unless the object was at a small airmass.

Some spectra were obtained at the $6.5 \mathrm{~m}$ MMT Observatory with the Blue Channel spectrograph (Schmidt et al. 
Table 2

Journal of Observations

\begin{tabular}{|c|c|c|c|c|c|c|c|c|c|c|c|c|c|c|}
\hline & UT Date $^{\mathrm{a}}$ & $\mathrm{HJD}^{\mathrm{b}}$ & Phase $^{c}$ & Tel. $^{\mathrm{d}}$ & $\begin{array}{c}\text { Range } \\
(\AA)\end{array}$ & $\begin{array}{c}\text { Res. }^{\mathrm{e}} \\
(\AA)\end{array}$ & $\begin{array}{l}\text { P.A. }{ }^{f} \\
\left({ }^{\circ}\right)\end{array}$ & $\begin{array}{c}\text { Par. }^{\mathrm{g}} \\
\left(^{\circ}\right)\end{array}$ & Air. & Flux Std. & $\begin{array}{c}\text { See. }^{\mathrm{i}} \\
\left({ }^{\prime \prime}\right)\end{array}$ & $\begin{array}{l}\text { Slit } \\
\left({ }^{\prime \prime}\right)\end{array}$ & $\begin{array}{c}\text { Exp. } \\
\text { (s) }\end{array}$ & Observer $(s)^{\mathrm{j}}$ \\
\hline \multicolumn{15}{|l|}{ SN 1997do } \\
\hline & 1997-11-02.45 & 2450754.95 & -11 & FLWO & $3720-7540.5$ & 7.0 & 90.0 & -128.7 & 1.07 & F34 & $1-2$ & 3 & 600 & DK \\
\hline & 1997-11-03.42 & 2450755.92 & -10 & FLWO & $3720-7540.5$ & 7.0 & 90.0 & -114.7 & 1.11 & F34 & 1 & 3 & 600 & DK \\
\hline & 1997-11-06.49 & 2450758.99 & -7 & FLWO & $3720-7540.5$ & 7.0 & 90.0 & -177.4 & 1.04 & F34 & $1-2$ & 3 & 1200 & $\mathrm{~PB}$ \\
\hline & 1997-11-07.49 & 2450760.00 & -6 & FLWO & $3720-7540.5$ & 7.0 & 90.0 & 172.3 & 1.04 & F34 & $1-2$ & 3 & 900 & PB \\
\hline & $1997-11-22.44$ & 2450774.95 & 8.5 & FLWO & $3720-7540.5$ & 7.0 & 90.0 & -172.3 & 1.04 & F34 & $1-2$ & 2 & $1200+900$ & $\mathrm{~PB}, \mathrm{JK}, \mathrm{BW}$ \\
\hline & $1997-11-24.44$ & 2450776.94 & 10.5 & FLWO & $3720-7540.5$ & 7.0 & 90.0 & -173.3 & 1.04 & $\mathrm{~F} 34$ & $1-2$ & 3 & 900 & PB \\
\hline & $1997-11-25.36$ & 2450777.87 & 11.5 & FLWO & $3720-7540.5$ & 7.0 & 90.0 & -116.2 & 1.09 & F34 & 2 & 3 & 1020 & $\mathrm{~PB}$ \\
\hline & $1997-11-26.36$ & 2450778.86 & 12.5 & FLWO & $3720-7540.5$ & 7.0 & 90.0 & -114.9 & 1.09 & $\mathrm{~F} 34$ & $2-3$ & 3 & 1200 & PB \\
\hline & $1997-11-28.48$ & 2450780.98 & 14.5 & FLWO & $3720-7540.5$ & 7.0 & 90.0 & 134.2 & 1.08 & F34 & $2-3$ & 3 & 1200 & PB \\
\hline & $1997-11-29.53$ & 2450782.04 & 15.5 & FLWO & $3720-7540.5$ & 7.0 & 90.0 & 104.7 & 1.21 & F34 & 1.5 & 3 & 720 & $\mathrm{~PB}$ \\
\hline & $1997-12-04.31$ & 2450786.82 & 20.5 & FLWO & $3720-7540.5$ & 7.0 & 90.0 & -105.6 & 1.15 & F34 & 2 & 3 & 900 & $\mathrm{JM}$ \\
\hline & $1997-12-05.28$ & 2450787.79 & 21.5 & FLWO & $3720-7540.5$ & 7.0 & 90.0 & -96.0 & 1.24 & F34 & 2 & 3 & 900 & $\mathrm{JM}$ \\
\hline
\end{tabular}

Notes.

For nights when multiple observations were taken at the same telescope, either as repeated exposures with the same setup or observations with different grating tilts, only a single observation is reported, with all relevant information described in this table representing the overall mid-point of the observations. When observations of the same SN were made on the same night with two different telescopes, we list them separately in this table, but only show a combined spectrum in the figures.

${ }^{a}$ UT at the midpoint of observation(s).

${ }^{\mathrm{b}}$ Heliocentric Julian date at the midpoint of observation(s).

${ }^{c}$ Phase of spectrum relative to $B$-band maximum, rounded to the nearest half day. The heliocentric Julian date for $B$-band maximum is taken from Table 4 of Jha et al. (2007) for all of the SNe except SN 2000cx, for which the date of $B$-band maximum from Li et al. (2001a) is used. The phase has also been corrected for relativistic time dilation using the recession velocity of the host galaxy as listed in Table 1 .

d Telescope used for this spectrum, FLWO = F. L. Whipple Observatory, MMTO = MMT Observatory.

e Approximate spectral resolution (full width at half maximum intensity).

${ }^{\mathrm{f}}$ Observed position angle during the observation(s).

$\mathrm{g}$ Average parallactic angle over the course of the observation(s).

${ }^{\mathrm{h}}$ Seeing is based upon estimates by the observers.

${ }^{\mathrm{i}}$ Standard stars: F25 = Feige 25, F34=Feige 34, F56 = Feige 56, F110 = Feige 110, H600 = Hiltner 600, BD33 = BD+33 2642 , BD28 $=$ BD+28 ${ }^{\circ} 4211-$ (Stone 1977; Massey et al. 1988; Massey \& Gronwall 1990); F66 = Feige 66-(Oke 1990); G191 = G191B2B, HZ44 = HZ 44, HZ14 = HZ 14-(Oke 1974; Massey et al. 1988); HD84 = HD 84937, BD26 = BD+26²606-(Oke \& Gunn 1983); HD217 = HD 217086—(Massey et al. 1988).

j Observers: $\mathrm{ZB}=\mathrm{Z}$. Balog, $\mathrm{PB}=\mathrm{P}$. Berlind, $\mathrm{AB}=\mathrm{A}$. E. Bragg, $\mathrm{NC}=\mathrm{N}$. Caldwell, $\mathrm{MC}=\mathrm{M}$. L. Calkins, BC $=\mathrm{B} . \mathrm{J} . \mathrm{Carter}, \mathrm{PC}=\mathrm{P} . \mathrm{Challis}, \mathrm{KD}=\mathrm{K} . \mathrm{Dendy}$ Concannon, EF = E. E. Falco, PG = P. M. Garnavich, GG = G. J. M. Graves, JH = J. P. Huchra, SJ = S. Jha, DK = D. M. Koranyi, JK = J. Kuraszkiewicz, $\mathrm{JM}=\mathrm{J} . \mathrm{A}$. Mader, $\mathrm{AM}=\mathrm{A}$. Mahdavi, $\mathrm{TM}=\mathrm{T}$. Matheson, $\mathrm{MP}=\mathrm{M}$. Phelps, $\mathrm{KR}=\mathrm{K}$. Rines, IS $=\mathrm{I}$. Song, $\mathrm{BW}=\mathrm{B}$. Wilkes.

(This table is available in its entirety in a machine-readable form in the online journal. A portion is shown here for guidance regarding its form and content)

1989). These observations were made during classically scheduled nights, not through queue-scheduled or interrupt time. The Blue Channel uses a $2688 \times 512$ UA/ITL CCD with a spatial scale of $0^{\prime \prime} .6$ per pixel in the binning mode used for these observations. The grating used yields a resolution of $\sim 8 \AA$.

\section{DATA REDUCTION}

The FAST data were all reduced in the same consistent manner. Using IRAF ${ }^{14}$, we would correct for overscan on the CCD frames and trim the extraneous portions. In general, the FAST CCD does not show a bias pattern in zero-time readouts, so we did not subtract bias frames to avoid introducing additional noise. In addition, dark current is not generally a problem with FAST. There are a few rare times after UV flashing that the chip has a dark-current problem, but it is bad enough that it cannot be corrected and so the affected portion of the spectrum has been trimmed off in the figures presented herein. The flat-field frames are combined and normalized with a low-order spline fit. The data are then flattened with these normalized flats. The spectra were optimally extracted using

\footnotetext{
14 IRAF is distributed by the National Optical Astronomy Observatory, which is operated by the Association of Universities for Research in Astronomy, Inc., under cooperative agreement with the National Science Foundation.
}

the prescription of Horne (1986) as implemented in the IRAF apall package. Wavelength calibration was accomplished with $\mathrm{HeNeAr}$ lamps taken immediately after each SN exposure. A low-order polynomial was fit to the lines in the calibration lamps, and the solution applied to the extracted objects. At a later stage in the reduction process, we applied small-scale adjustments derived from night-sky lines in the $\mathrm{SN}$ frames.

Once the data were extracted as one-dimensional, wavelength-calibrated spectra, we used our own routines in IDL to flux calibrate them. This entailed spline fits to the standard stars to assign fluxes. The relative spectrophotometry is good (see the discussion below), but no attempt was made to put the spectra on an absolute scale. Spectrophotometric standard stars used for each spectrum are listed in Table 2. Using the well-exposed continua of the spectrophotometric standard stars as smooth-spectrum sources, we removed telluric features from the spectra using techniques described by Wade \& Horne (1988), Bessell (1999), Matheson et al. (2000a).

The spectra presented here that were obtained in 19971999 were, in general, not observed at the parallactic angle (Filippenko 1982). Most spectra were taken at a small airmass, but some could be affected by atmospheric dispersion. Table 2 lists the observed position angle as well as the proper parallactic angle for each spectrum along with the airmass. Data taken for 


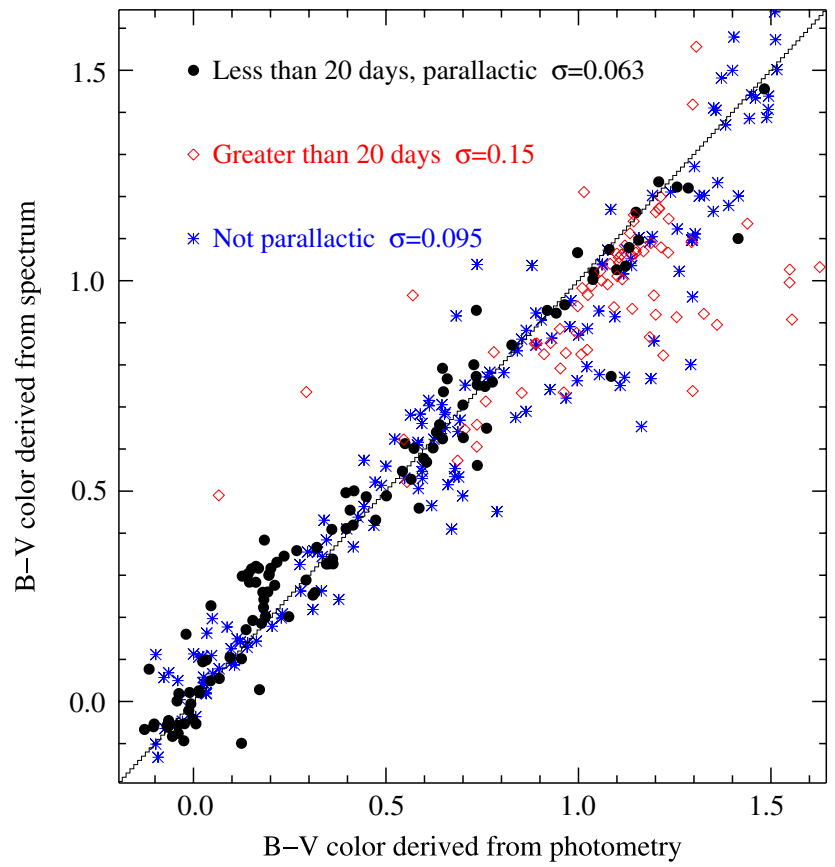

Figure 4. Comparison of the colors of the SNe Ia in our sample derived from photometry and spectroscopy. For objects observed at the parallactic angle (or small airmass) and within 20 days of $B$-band maximum (filled, black circles), the scatter around zero difference is 0.063 . For objects observed over 20 days after maximum (open, red diamonds), the scatter is 0.15 . For the early objects in our sample, when we did not consistently observe at the parallactic angle (blue asterisks), the scatter is 0.095 .

(A color version of this figure is available in the online journal)

the CfA SN group with FAST from 2000 on were observed at the parallactic angle (unless at small airmass).

During the period that the observations described here were made, the optics of the FAST spectrograph blocked blue light, meaning that the FAST spectra do not suffer from second-order light contamination. ${ }^{15}$ The spectra obtained with the Blue Channel spectrograph can suffer from second-order contamination, but, through careful cross-calibration with standard stars of different colors, we have minimized the problems this might cause. On any given night, we would try to observe a relatively blue standard star (typically an sdO) and a relatively red standard star (typically an $\mathrm{sdF}$ ). The sdO standards provide a better calibration in the blue portion of our spectrum (below $\sim 4500 \AA$ ) where they will generally have more counts, but also lack the Balmer jump that can adversely affect calibration. The red standard stars will have little blue flux, and thus little second-order contamination. Most of our targets, even at relatively early phases when the spectra can be blue, have little second-order contamination as well. Each spectrum is calibrated with both the blue and the red standard stars. The blue and red portions of each spectrum are then joined, typically near $4500 \AA$, so that we get a good calibration of the blue half, without suffering second-order problems in the red half. Some residual contamination remains, but tests with standard stars indicate that we have mitigated most of the problems.

Because the spectra were selected from a sample for which calibrated light curves exist, we are able to check the accuracy of the relative spectrophotometry. We used the light curves of Jha

15 When we moved the grating tilt to observe at red wavelengths, we did use order-blocking filters.

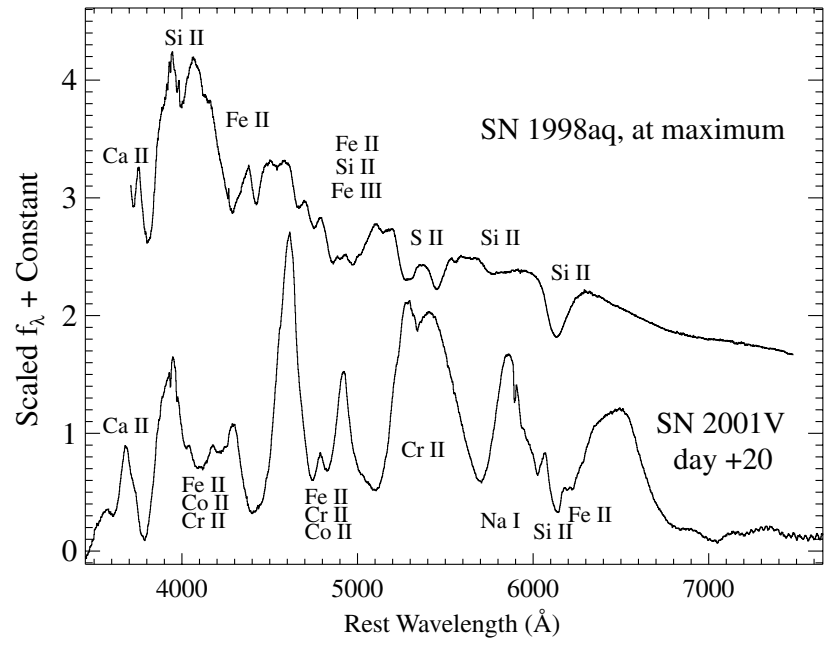

Figure 5. Spectrum of SN 1998aq at $B$-band maximum and spectrum of $\mathrm{SN}$ $2001 \mathrm{~V}$ at 20 days past $B$-band maximum. The flux units are $f_{\lambda}\left(\mathrm{erg} \mathrm{s}^{-1} \mathrm{~cm}^{-2}\right.$ $\AA^{-1}$ ) that have been normalized and then additive offsets applied for clarity. The systemic heliocentric velocity listed in Table 1 has been removed. Major features of the spectra are labeled.

et al. (2006) to determine the $B$ and $V$ magnitudes of the $\mathrm{SNe}$ at the time of each spectrum. When the photometry was not coincident, we interpolated from the nearest data points. We then took each spectrum and convolved it with $B$ and $V$ filter functions in order to derive a $B-V$ color. Figure 4 shows the comparison between the $B-V$ color based on photometry and the $B-V$ color derived from the spectra. For the objects observed from 1997 to late 1999 when we did not consistently use the parallactic angle, the scatter around zero difference in color is $\sigma=0.095$. The spectra observed at phases later than 20 days past $B$-band maximum also have a relatively high scatter of $\sigma=0.15$. There are a number of factors that could cause this difference. One is that the spectrum becomes increasingly dominated by line emission as it ages, so that convolution with a filter that is not precisely matched to the photometry might introduce a systematic error. Another important difference with the later spectra is that they are fainter, so that host galaxy contamination becomes more significant. When the spectra were observed at the parallactic angle during phases when the spectrum was more continuum-dominated, the scatter is only $\sigma=0.063$, a relatively small error. We believe this indicates how well-calibrated the spectra in this sample are.

In Figure 5, we show two examples of SNe Ia spectra from the sample. SN 1998aq is shown at $B$-band maximum, while SN $2001 \mathrm{~V}$ is shown at 20 days past $B$-band maximum. Both of these SNe are what would be considered photometrically normal with SN 1998aq having a $\Delta m_{15}(B)$ of 1.13 (Riess et al. 2005) and SN 2001V having a $\Delta m_{15}(B)$ of 0.99 (K. Mandel et al. 2007, in preparation). These spectra are fairly typical results for the brighter SNe. In addition, we label some major features of the spectra in order to facilitate discussion of the individual objects below (see, e.g., Branch et al. 2005). To demonstrate the differences among the spectra of SNe with different light-curve shapes, we show two extreme examples compared with the more normal SN 1998aq in Figure 6. SN 1999aa has a $\Delta m_{15}(B)$ of 0.85 and was overluminous. Note the weaker Si II and stronger Fe III. In contrast, SN 1999 by has a $\Delta m_{15}(B)$ of 1.90 and was subluminous. It shows stronger Si II and Ti II. 


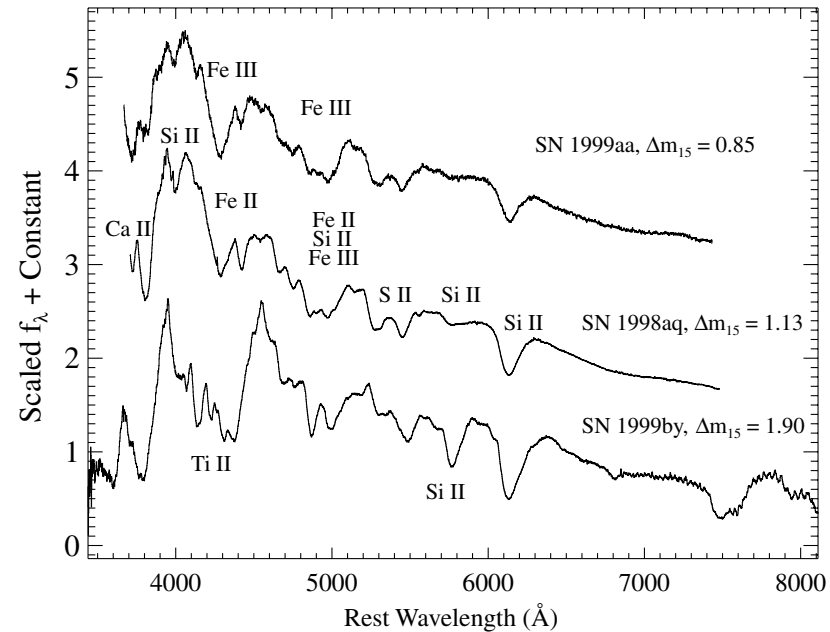

Figure 6. Spectra of SNe 1998aq, 1999aa, and 1999by at $B$-band maximum The flux units are $f_{\lambda}\left(\mathrm{erg} \mathrm{s}^{-1} \mathrm{~cm}^{-2} \AA^{-1}\right)$ that have been normalized and then additive offsets applied for clarity. The systemic heliocentric velocity listed in Table 1 has been removed. Major features of the spectra are labeled. Note that the overluminous SN 1999aa has weaker Si II than SN 1998aq and stronger Fe III. The underluminous SN 1999by has stronger Si II (especially near $5800 \AA$ ) and Ti II.

\section{COMMENTS ON INDIVIDUAL SUPERNOVAE}

SN 1997do. This SN was discovered on 1997 October 31 during the course of the Beijing Astronomical Observatory (BAO) SN survey (Qiu et al. 1997). A spectrum obtained by Qiu et al. on 1997 November 1 showed that SN 1997do was of Type Ia. The CfA spectra (Figure 7) begin 11 days before $B$-band maximum and continue for three weeks past maximum.

For Figures 8-44, the flux units, wavelength scale, and epoch for each spectrum are as described in Figure 7.

SN 1997dt. Another product of the BAO SN survey, this SN was discovered on 1997 November 22 (Qiao et al. 1997). Qiao et al. also reported that a spectrum taken the same night as the discovery indicated that SN $1997 \mathrm{dt}$ was of Type Ia. The CfA spectra (Figure 8 ) begin 10 days before $B$-band maximum, with good coverage through 2 days past the time of maximum. There is some galaxy contamination in the spectra, as shown by the strong narrow emission lines apparent in the spectra.

SN 1998V. This SN was discovered by the U.K. Nova/Supernova Patrol on 1998 March 10 (Hurst et al. 1998b). It was classified as an SN Ia (Jha et al. 1998d) based up the first spectrum in the CfA sample (Figure 9), obtained at maximum. We have some coverage of the post-maximum decline and later phases.

SN 1998ab. The BAO SN survey also found SN 1998ab on 1998 April 1 (Wei \& Li 1998). The first CfA spectrum (Figure 10) was used to report the Type of the $\mathrm{SN}$ as Ia (Garnavich et al. 1998b). This spectrum was obtained 8 days before maximum. In addition, Garnavich et al. noted that the Si II feature was not apparent in the spectrum, but absorptions associated with Fe III were present, indicating that this was a spectroscopically peculiar SN similar to SN 1991 T (Filippenko et al. 1992a; Phillips et al. 1992) at early epochs. Unfortunately, we were not able to obtain more spectra of this object in the photospheric phase, but there is a large amount of coverage at several months past $B$-band maximum.

SN 1998aq. Another SN discovered by the U.K. Nova/Supernova Patrol, SN 1998aq was found on 1998 April 13

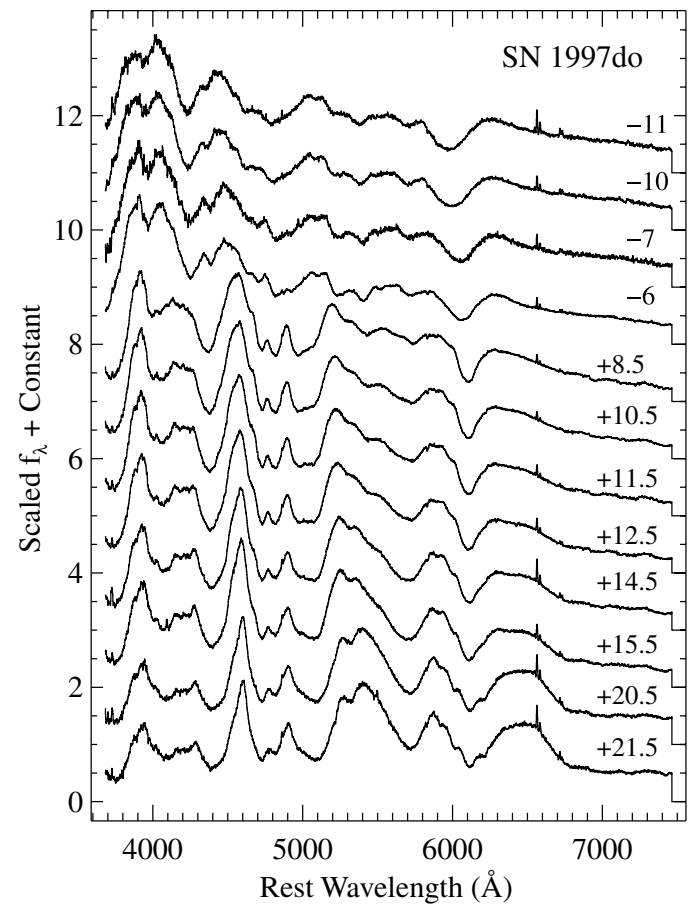

Figure 7. Spectra of SN 1997do. The flux units are $f_{\lambda}\left(\mathrm{erg} \mathrm{s}^{-1} \mathrm{~cm}^{-2} \AA^{-1}\right)$ that have been normalized and then additive offsets applied for clarity. The zero-flux level for each spectrum is marked with an extension on the red edge of the spectrum (occasionally, this is marked on the blue edge if that produces a clearer presentation). The systemic heliocentric velocity listed in Table 1 has been removed. The numbers associated with each spectrum indicate the epoch of the spectrum relative to $B$-band maximum.

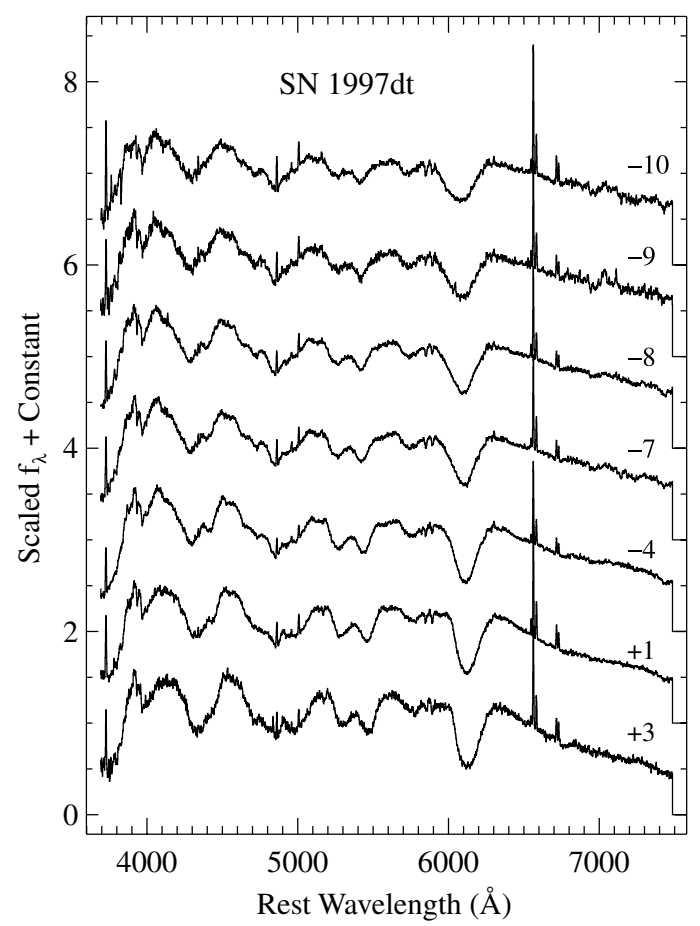

Figure 8. Spectra of SN 1997dt.

(Hurst et al. 1998a). Ayani \& Yamaoka (1998) and Garnavich et al. (1998c) reported that the spectrum showed SN 1998aq to be of Type Ia. Ayani \& Yamaoka also felt that the strength 


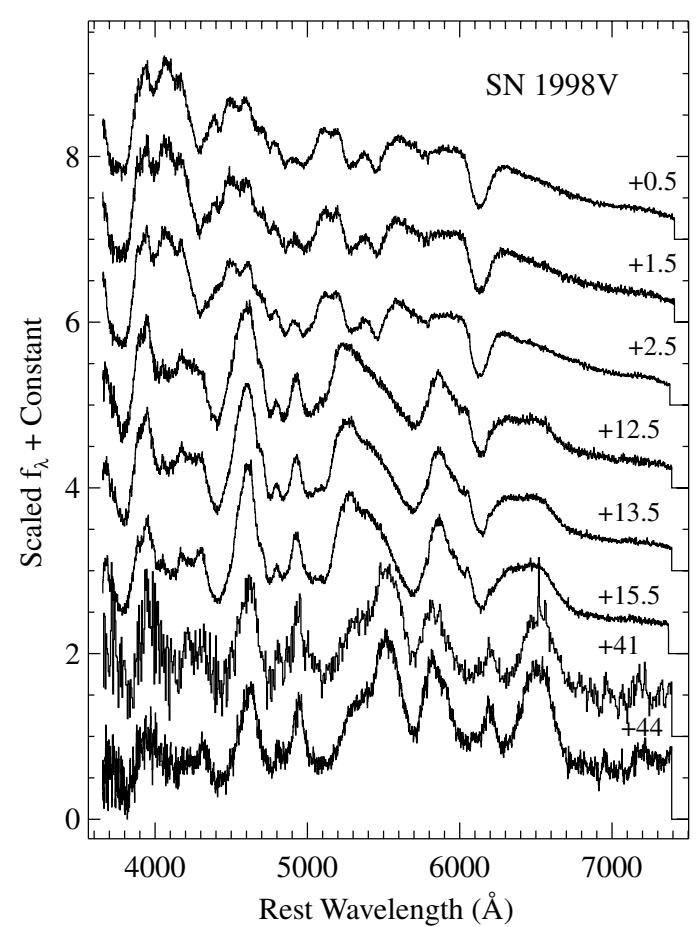

Figure 9. Spectra of SN 1998V. The day +41 spectrum has been rebinned for clarity.

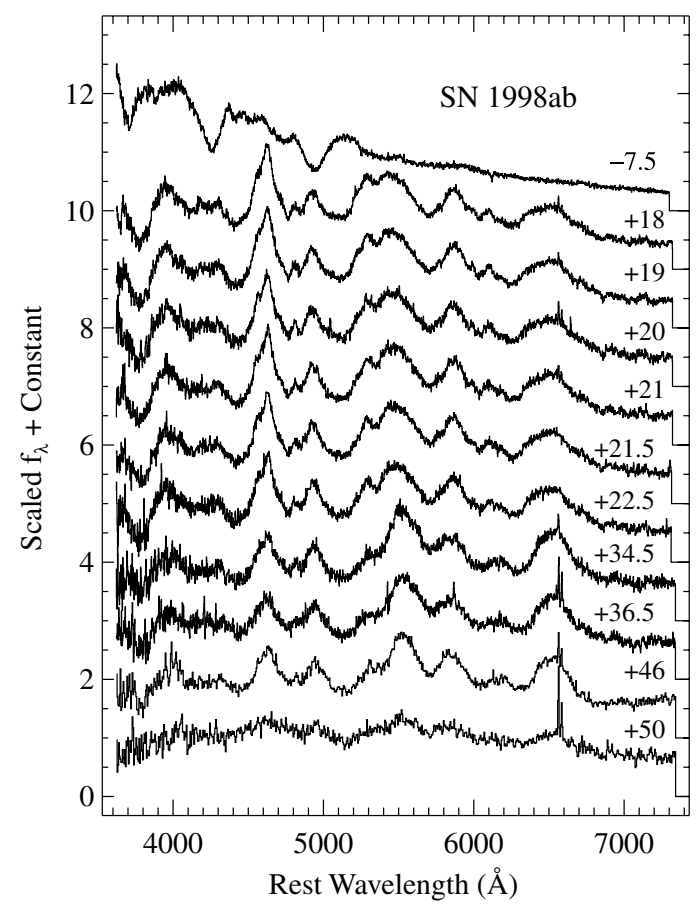

Figure 10. Spectra of SN 1998ab. The days $+36.5,+46$, and +50 spectra have been rebinned for clarity.

of the Si II $\lambda 5800$ line might indicate that SN 1998aq was subluminous, but the full set of CfA spectra (Figures 11 and 12) shows that it was spectroscopically normal. Although there are no pre-maximum spectra, there is good coverage starting with the first spectrum taken 1 day past maximum, continuing with almost daily spectra in the weeks past maximum. There is an

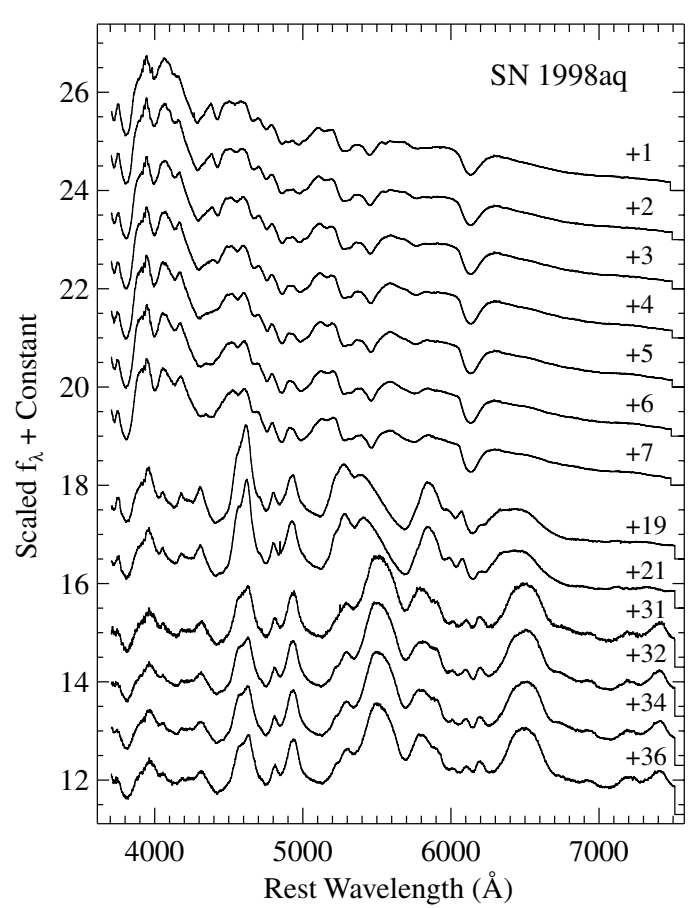

Figure 11. Early spectra of SN 1998aq.

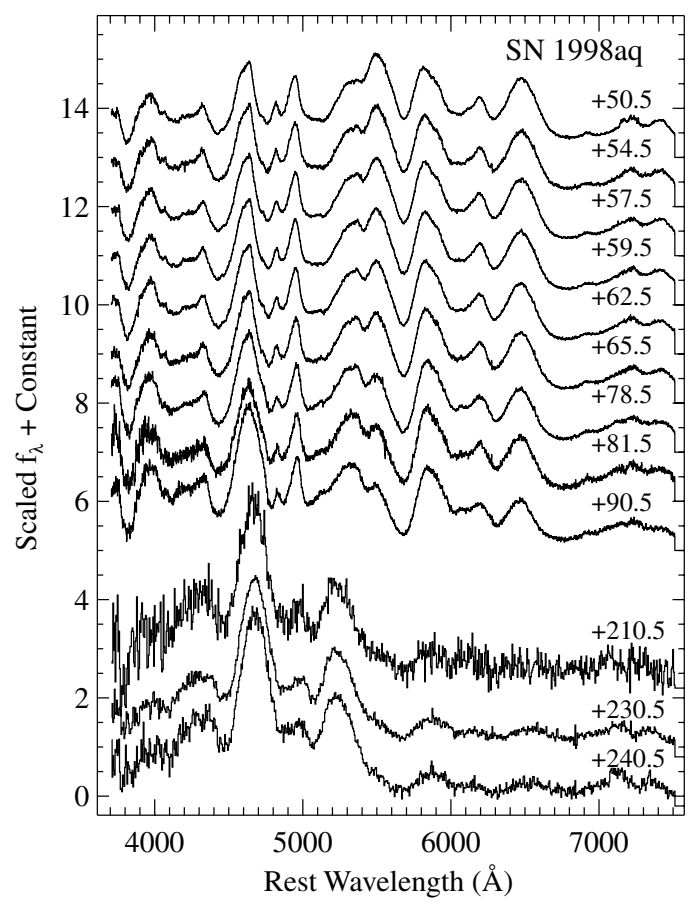

Figure 12. Late spectra of SN 1998aq.

extensive set of spectra obtained several weeks past maximum, as well as a few in the nebular phase. Some of these spectra have been published and analyzed by Branch et al. (2003), but we include them here for completeness.

SN 1998bp. This SN was also found by the U.K. Nova/Supernova Patrol on 1998 April 29 (Hurst \& Armstrong 1998). A spectrum taken the next night showed that is was of Type Ia, but possibly spectroscopically peculiar (Patat \& Maia 1998). The first CfA spectrum (Figure 13, obtained 3 days 


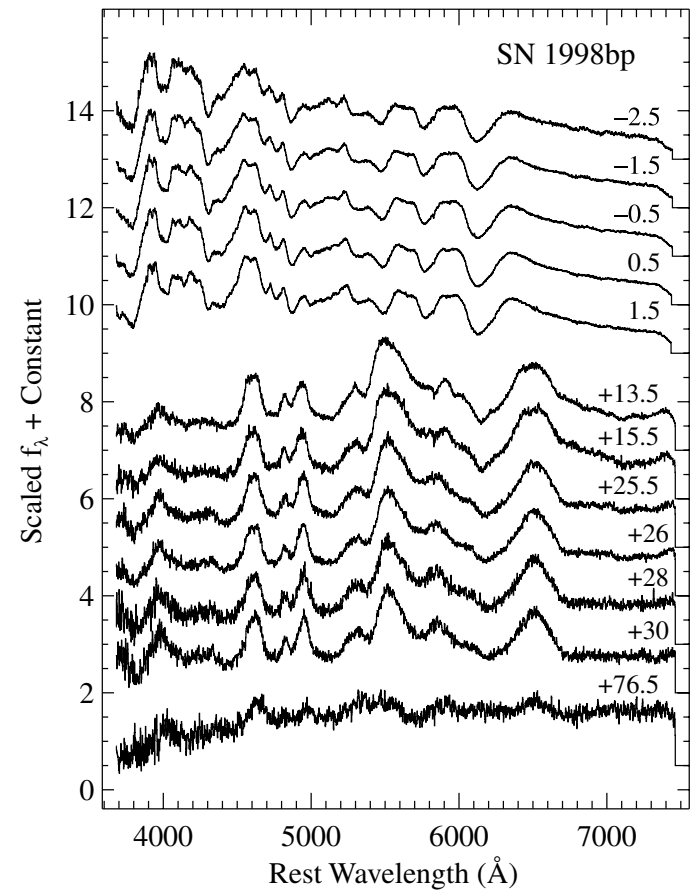

Figure 13. Spectra of SN 1998bp.

before maximum) also prompted a report that the object seemed peculiar (Jha et al. 1998b), specifically that the Si II $\lambda 5800$ line was strong. As seen in Figure 13, the strength of the $\lambda 5800$ feature is similar to what is observed in subluminous SNe Ia (e.g., SN 1991bg Filippenko et al. 1992b; Leibundgut et al. 1993), but the blue half of the spectrum appears more normal. The $\Delta m_{15}(B)$ value of 1.83 from Jha et al. (2006) confirms that this was a peculiar SN Ia. The CfA sample has good coverage near maximum, as well as several at a few weeks past maximum.

SN 1998bu. This SN was discovered by Villi (1998) on 1998 May 9. Two groups reported that spectroscopy indicated that SN 1998bu was of Type Ia (Ayani et al. 1998). The CfA sample of spectra is large (Figures 14 and 15), with good coverage near maximum (the first being 3 days before maximum), as well as many spectra up to two months past maximum and few in the nebular phase. Some of the CfA spectra were shown and discussed by Jha et al. (1999c), but the spectra presented herein were rereduced to be consistent with the rest of this spectroscopic sample. This was a bright SN that was followed extensively by many groups (e.g., Suntzeff et al. 1999).

SN 1998de. This SN was found in the course of the Lick Observatory Supernova Search (LOSS) on 1998 July 23 (Modjaz et al. 1998a). The initial classification as a Type Ia was based upon our first CfA spectrum (Figure 16) obtained 7 days before maximum. This SN also appeared to be spectroscopically peculiar, with a strong Si II $\lambda 5800$ line and prominent Ti II absorptions in the blue (Garnavich et al. 1998d). This characterization as a subluminous SN Ia was confirmed photometrically and spectroscopically by Modjaz et al. (2001) (and the $\Delta m_{15}(B)$ value of 1.93 from Jha et al. 2006) and can be seen in the series of spectra of Figure 16.

SN 1998dh. Another product of the LOSS, SN 1998dh was found on 1998 July 20 ( $\mathrm{Li}$ et al. 1998). The first CfA spectrum (Figure 17), obtained 9 days before maximum, was used to report that it was an SN Ia (Garnavich et al. 1998a). There

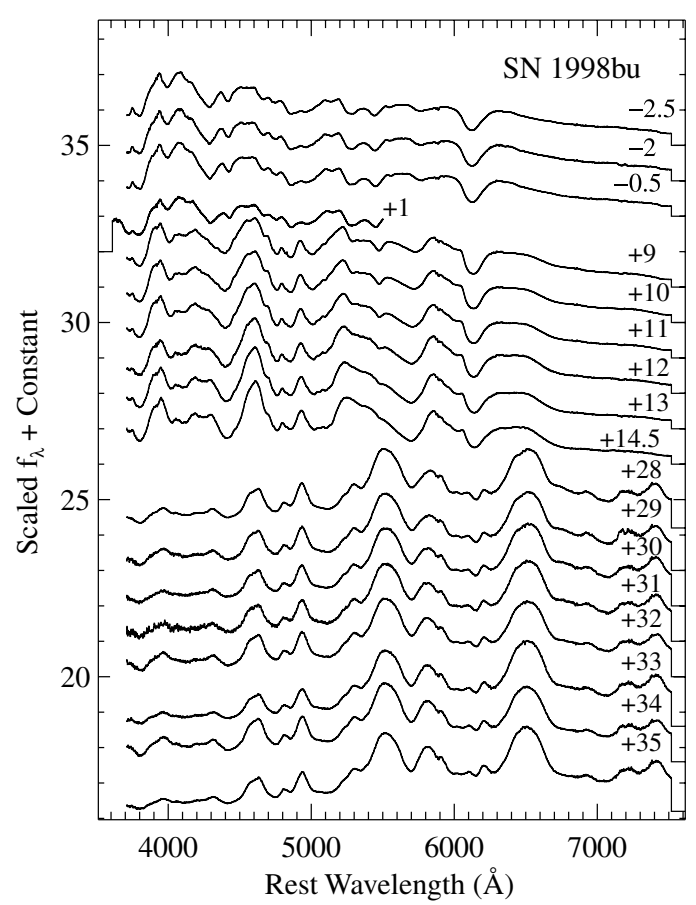

Figure 14. Early spectra of SN 1998bu.

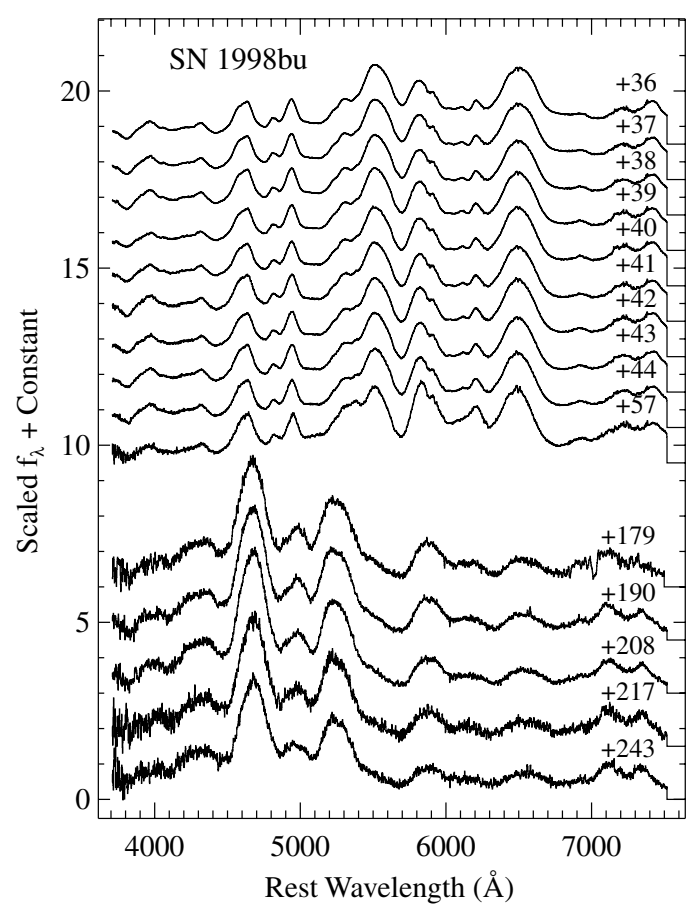

Figure 15. Late spectra of SN 1998bu.

is good coverage before maximum, as well as some later coverage.

SN 1998dk. The LOSS also discovered SN 1998dk on 1998 August 19 (King et al. 1998). It was classified as an SN Ia (Filippenko \& De Breuck 1998). The telescopes on Mt. Hopkins are traditionally closed during August for the Arizona monsoon season, so the CfA spectra begin 10 days after maximum (Figure 18), but with several spectra in the weeks past maximum. 


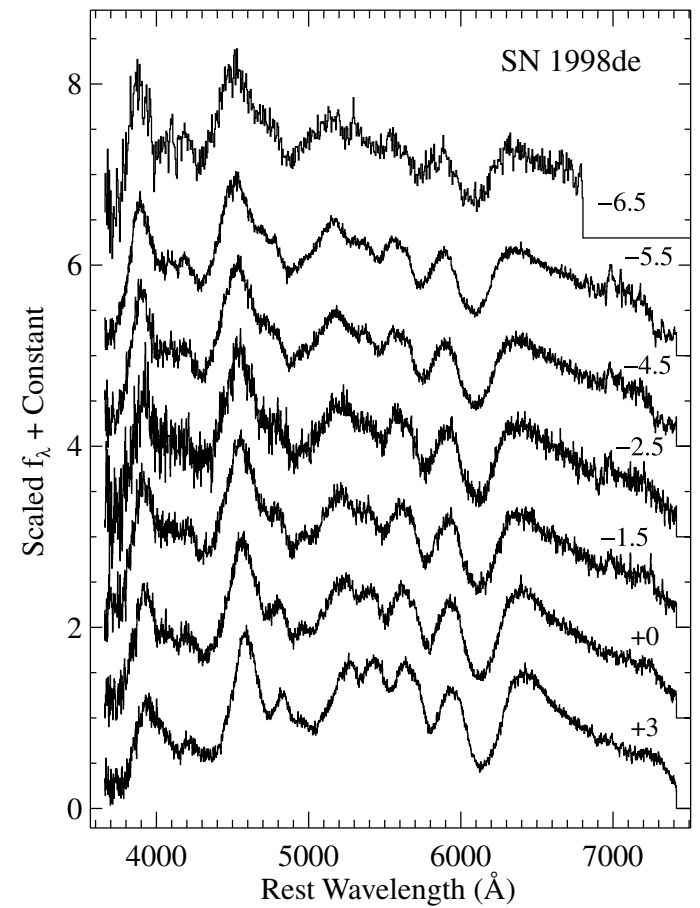

Figure 16. Spectra of SN 1998de. The day -6.5 spectrum has been trimmed and rebinned for clarity.

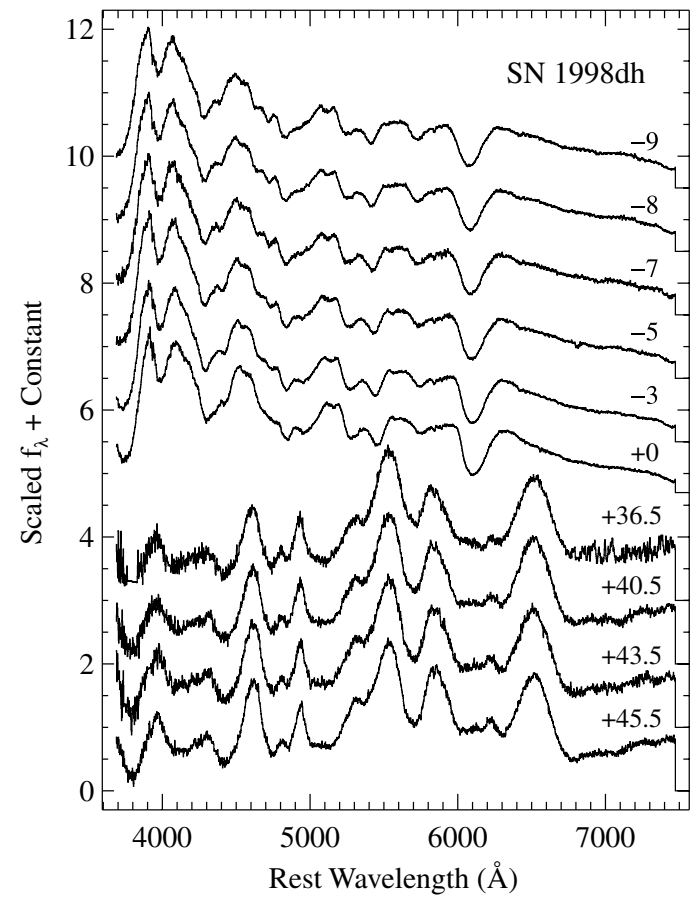

Figure 17. Spectra of SN 1998dh.

SN 1998dm. This SN was also found by LOSS on $1998 \mathrm{Au}-$ gust 22 (Modjaz et al. 1998b), and SN 1998dm was subsequently classified as an SN Ia (Filippenko \& De Breuck 1998). Filippenko \& De Breuck noted that the spectra were unusually red and that the Si II $\lambda 5800$ line seemed relatively strong, suggesting that this was a subluminous event. Our spectra (Figure 19) appear more normal (although our early coverage was limited by the August shutdown described above, leading to

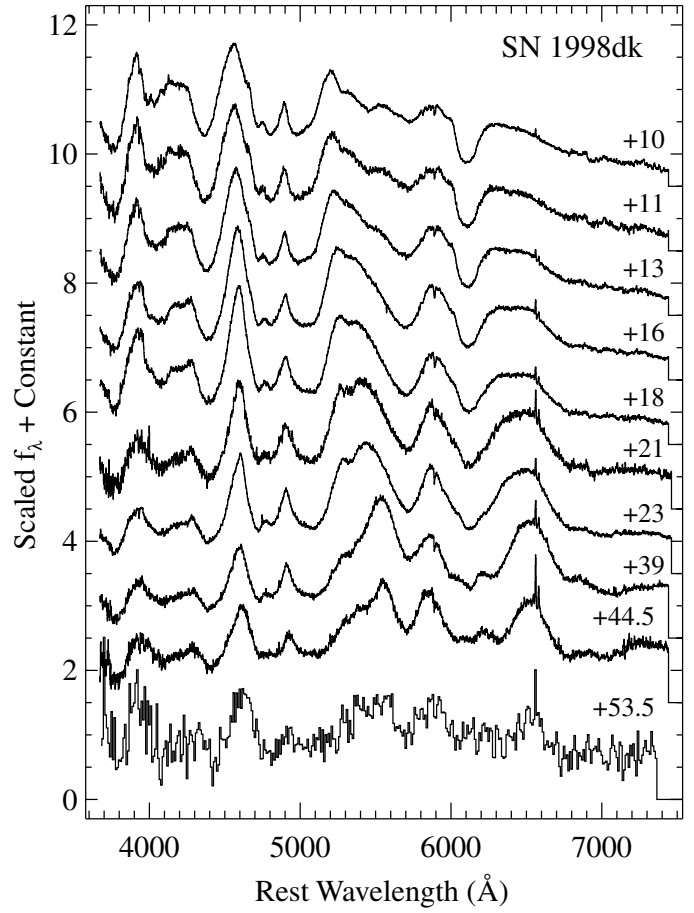

Figure 18. Spectra of SN $1998 \mathrm{dk}$. The day +53.5 spectrum has been rebinned for clarity.

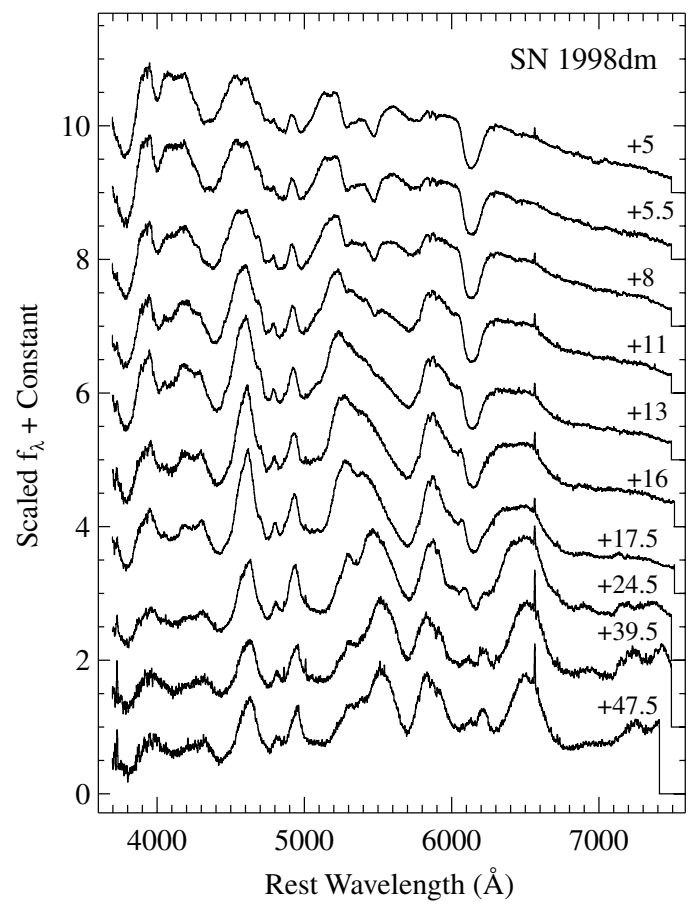

Figure 19. Spectra of SN 1998dm.

the earliest CfA spectrum being 5 days past maximum), and the $\Delta m_{15}(B)$ value of 1.07 (Jha et al. 2006) confirms that SN $1998 \mathrm{dm}$ was not clearly peculiar.

SN 1998ec. The BAO supernova survey found SN 1998ec on 1998 September 26 (Qiu et al. 1998). Our first CfA spectrum (Figure 20), observed 3 days before maximum, was used to classify it as an SN Ia (Jha et al. 1998a). This object has a more 


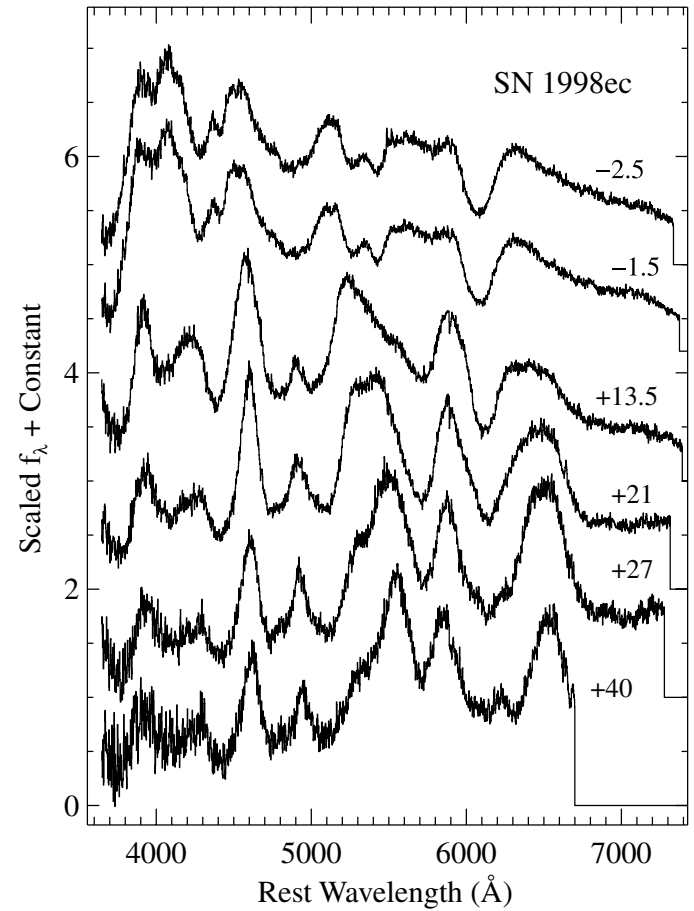

Figure 20. Spectra of SN 1998 ec. The day +40 spectrum has been trimmed for clarity.

limited number of spectra, but the pre-maximum observations are valuable.

SN 1998eg. Another product of the U.K. Supernova Patrol, SN 1998eg was found on 1998 October 19 (Hurst et al. 1998c). Two groups (including the CfA SN group using our first spectrum obtained at maximum) separately classified it as an SN Ia (Salvo et al. 1998). Our spectra cover the period from maximum to a few weeks past maximum (Figure 21).

SN 1998es. The LOSS discovered SN 1998es on 1998 November 13 (Halderson et al. 1998). Our first CfA spectrum (Figure 22) was used to classify it as an SN Ia (Jha et al. 1998c). Jha et al. noted that the Si II $\lambda 6355$ feature was relatively weak and that the spectrum overall resembled that of SN 1991T (Filippenko et al. 1992a; Phillips et al. 1992) at early epochs. The $\Delta m_{15}(B)$ value of 0.87 (Jha et al. 2006) also indicates that this was a 91T-like event. The CfA sample (Figures 22 and 23) begins at 10 days before maximum, and continue with good coverage until just past maximum. There is also a large set of spectra covering several few weeks past maximum.

SN 1999X. This SN was found by Schwartz (1999) on 1999 January 27. Our first CfA spectrum (Figure 24), obtained 12 days after maximum, was used to classify SN 1999X as an SN Ia (Garnavich et al. 1999a). The spectra cover two to four weeks past maximum.

SN 1999aa. Arbour \& Armstrong (1999) found SN 1999aa on 1999 February 11 (independent discoveries were also reported by Qiao et al. 1999 and Nakano \& Kushida 1999). A spectrum obtained the next day revealed that SN 1999aa was a spectroscopically peculiar SN Ia (Filippenko et al. 1999). The spectrum had some similarities to SN 1991T (Filippenko et al. 1992a; Phillips et al. 1992), with weak Si II $\lambda 6355$ and absorptions due to Fe III. One difference was an absorption near $3750 \AA$. This feature (Ca II H, and $\mathrm{K}$ ) was not present in SN 1991T. The $\Delta m_{15}(B)$ value of 0.85 (Jha et al. 2006) shows

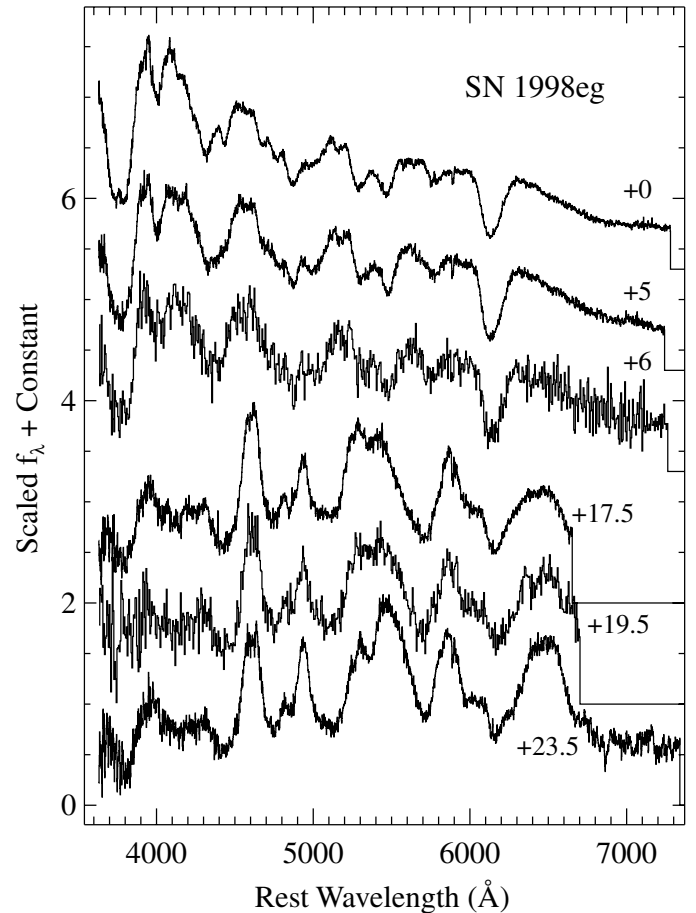

Figure 21. Spectra of SN 1998eg. The days +5 and +19.5 spectra have been rebinned and the days +17.5 and +19.5 spectra have been trimmed for clarity.

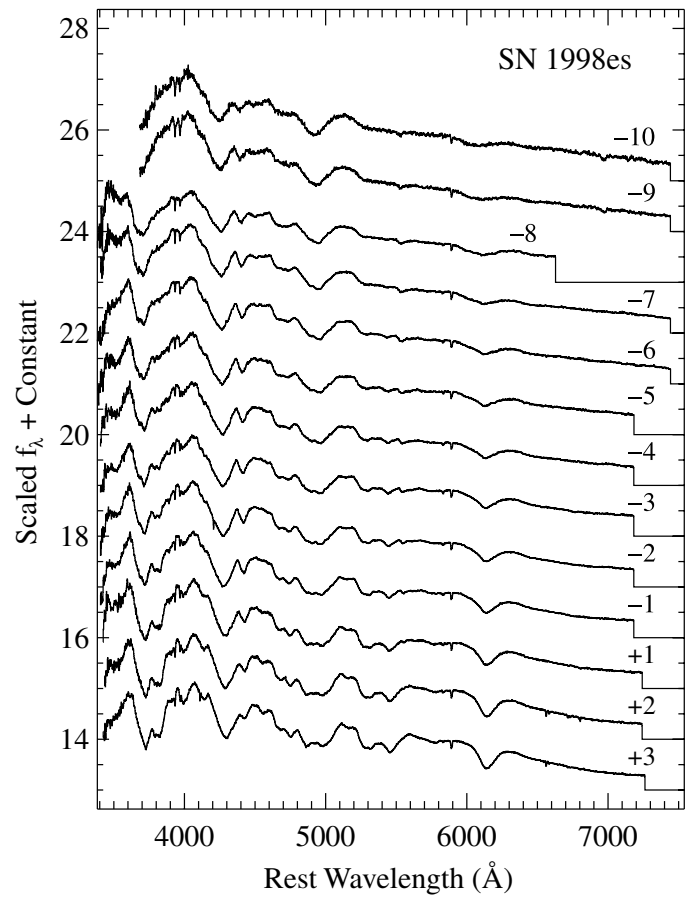

Figure 22. Early spectra of SN 1998es.

that it is similar to SN $1991 \mathrm{~T}$ photometrically as well. The CfA spectra have extensive coverage from 9 days before maximum through 3 months past maximum (Figures 25 and 26). This bright and unusual SN was well observed by many groups (e.g., Li et al. 2001b; Garavini et al. 2004).

SN 1999ac. This SN was found by the LOSS on 1999 February 26 (Modjaz et al. 1999). A spectrum obtained by 


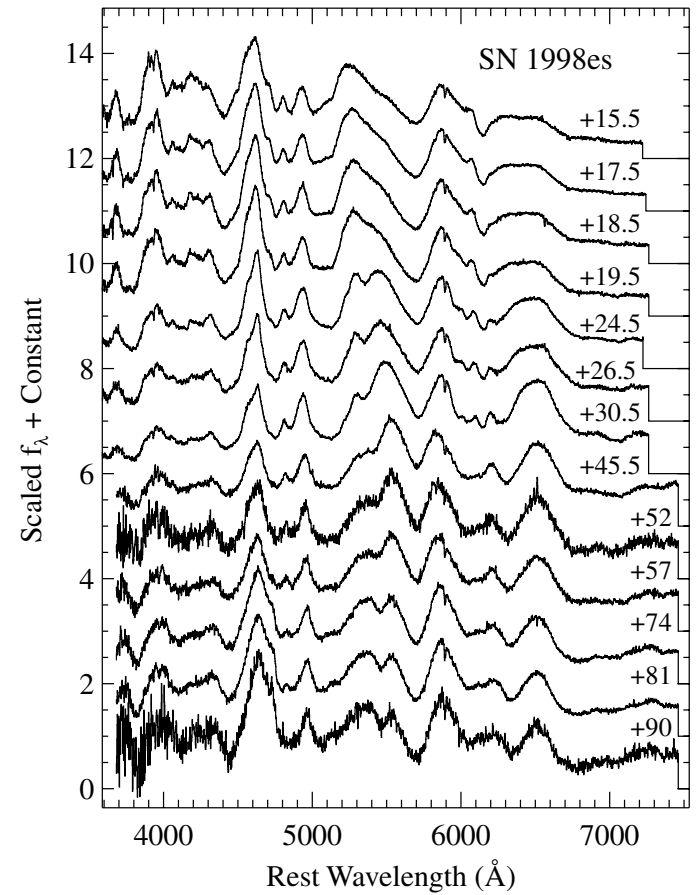

Figure 23. Late spectra of SN 1998es.

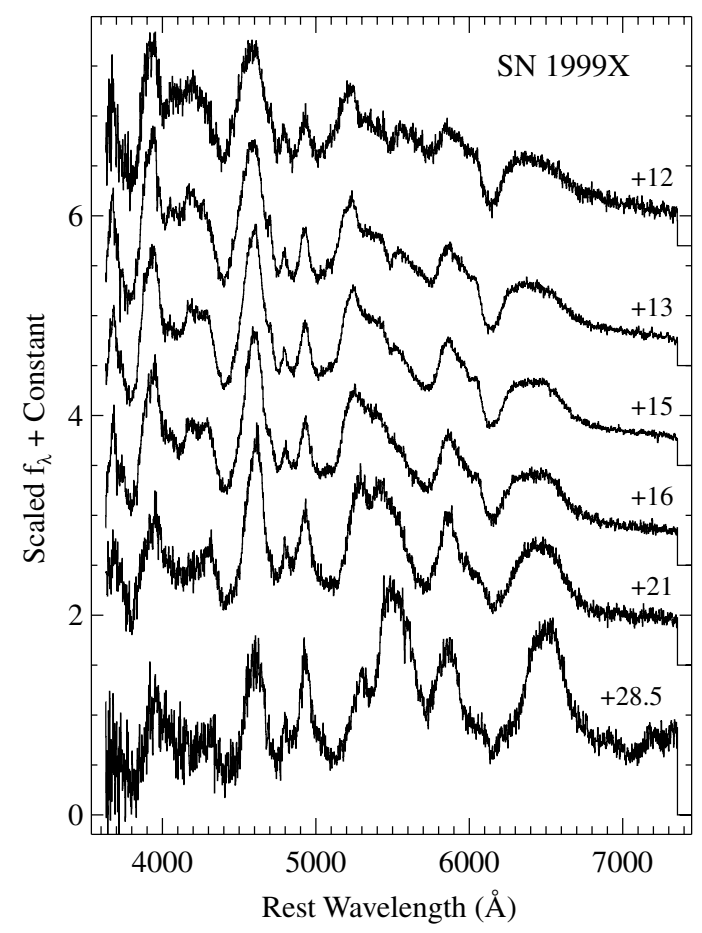

Figure 24. Spectra of SN 1999X.

Phillips et al. (1999) showed that it was strikingly similar to SN 1999aa. The CfA spectra of SN 1999ac have some near maximum (the first at 4 days before maximum) and a large number one to three months past maximum (Figure 27).

SN 1999by. Both the LOSS and the U.K. Supernova Patrol independently discovered SN 1999by on 1999 April 30 (Arbour et al. 1999). Gerardy \& Fesen (1999) classified it as an SN Ia, and Garnavich et al. (1999b) reported that the spectra showed

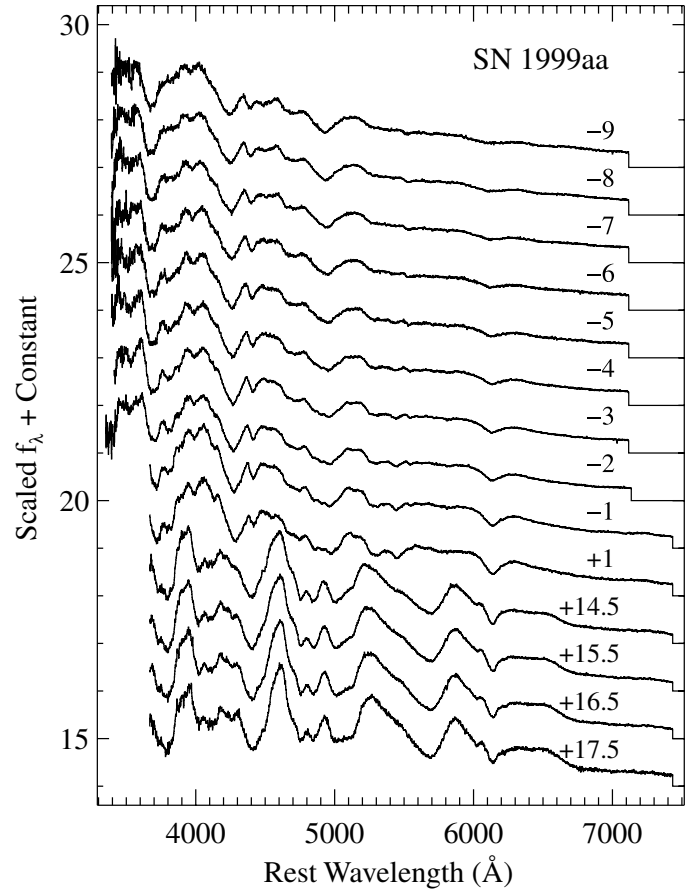

Figure 25. Early spectra of SN 1999aa.

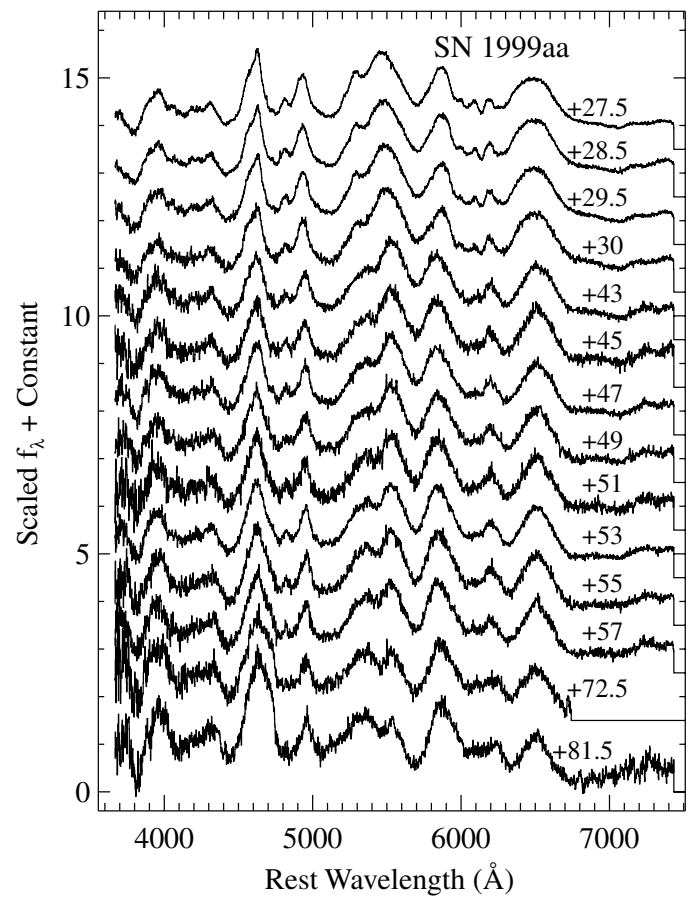

Figure 26. Late spectra of SN 1999aa.

some signs of peculiarity. The Si II $\lambda 5800$ line was relatively strong and Ti II features were apparent indicating that this was a subluminous SN Ia. This was confirmed photometrically (Garnavich et al. 2004) with a $\Delta m_{15}(B)$ of 1.90 . The CfA spectra have good coverage from 5 days before maximum through several weeks past maximum (Figure 28). Most of the spectra were shown and analyzed by Garnavich et al. (2004), but the spectra presented herein were rereduced to be consistent with the rest of this spectroscopic sample. This bright and peculiar 


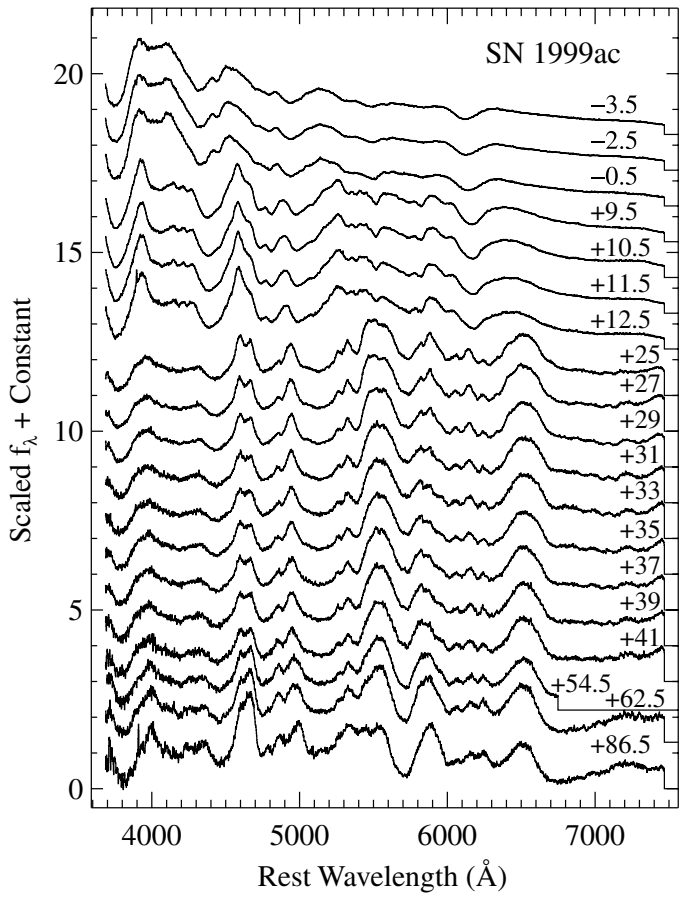

Figure 27. Spectra of SN 1999ac.

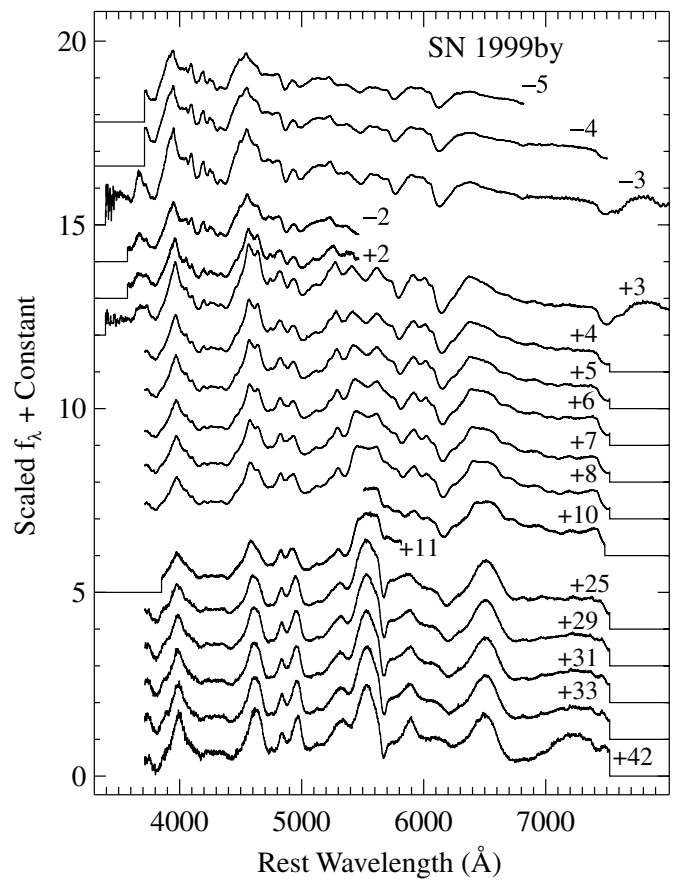

Figure 28. Spectra of SN 1999by.

SN was well observed by many groups, including Toth \& Szabó (2000), Vinkó et al. (2001), Howell et al. (2001), Höflich et al. (2002), and Garnavich et al. (2004).

SN 1999cc. Schwartz (1999) discovered SN 1999cc on 1999 May 8. Our first CfA spectrum (Figure 29), obtained 3 days before maximum showed that it was an SN Ia (Garnavich et al. 1999c). Our set of spectra includes a few near maximum and a few three weeks after maximum.

SN 1999cl. This SN was found by the LOSS on 1999 May 29 (Papenkova et al. 1999). Our first CfA spectrum

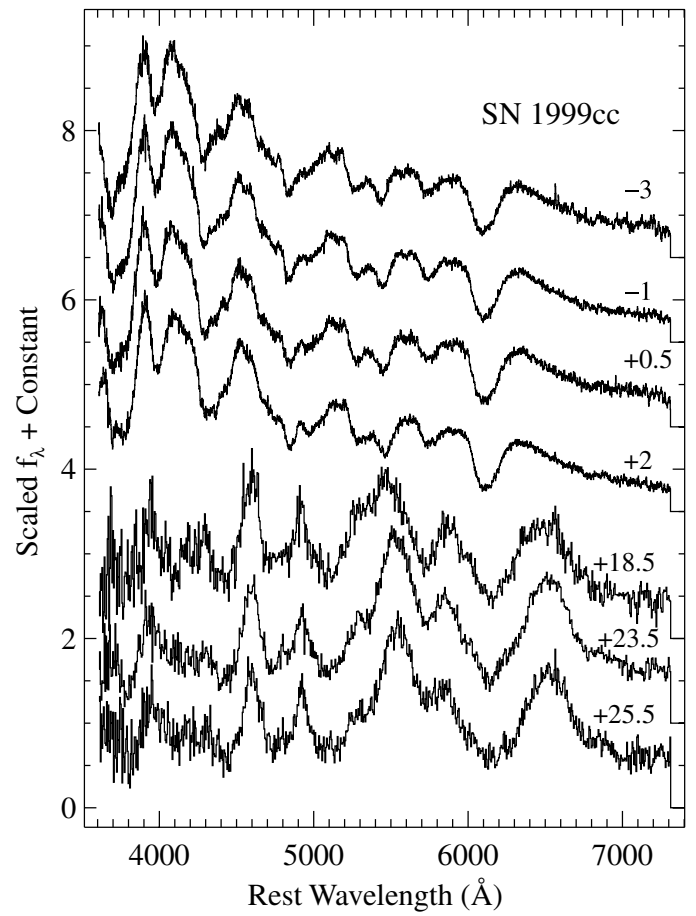

Figure 29. Spectra of SN 1999cc.

(Figure 30) indicated that it was of Type Ia, but there was some evidence of peculiarity (Garnavich et al. 1999d). The spectrum declined to the blue, there was a strong $\mathrm{Na}$ I $\mathrm{D}$ absorption at the host galaxy's velocity (equivalent width [EW] of $3.3 \AA$ ), and there was a relatively strong Galactic Na I D absorption (EW of $0.7 \AA$ ). These facts suggest that the $\mathrm{SN}$ was heavily extinguished by dust. In addition, the absorption features of the SN itself were broad, with the two components of the $\mathrm{S}_{\text {II }}$ "W" feature blended together. Our spectra cover from 8 days before to 9 days after maximum, as well as one spectrum about five weeks past maximum. The $\Delta m_{15}(B)$ value of 1.19 (Jha et al. 2006) shows that the faint, reddened nature of this $\mathrm{SN}$ is mainly caused by the intervening extinction.

SN 1999dq. Another LOSS discovery, SN 1999dq was found on 1999 September 5 ( $\mathrm{Li}$ 1999a). The spectrum was classified as an SN Ia, but with some peculiarities (Jha et al. 1999b). The Si II $\lambda 6355$ line was shallow and absorptions due to Fe III were present. Overall, there was strong similarity to SN 1991T (Filippenko et al. 1992a; Phillips et al. 1992). The spectrum also showed Na I D absorption at the host galaxy's velocity (EW of $1.5 \AA$ ) as well as an Na I D absorption of Galactic origin (EW of $0.8 \AA$ ), suggesting that the SN suffered from reddening. The CfA spectra (Figures 31 and 32) start at 10 days before maximum and continue almost daily until a week past maximum. There are also many spectra one to three months past maximum.

SN 1999ej. The LOSS also found SN 1999ej on 1999 October 18 (Friedman et al. 1999). Based upon our first CfA spectrum (Figure 33), obtained 1 day before maximum, it was classified as an SN Ia (Jha et al. 1999a). We have a few spectra, from near maximum to two weeks after maximum.

SN 1999gd. The LOSS discovered SN 1999gd on 1999 November 24 (Li 1999b). A spectrum revealed that it was of Type Ia and that a strong narrow Na I D absorption was present, implying that the $\mathrm{SN}$ was extinguished by dust (Filippenko 


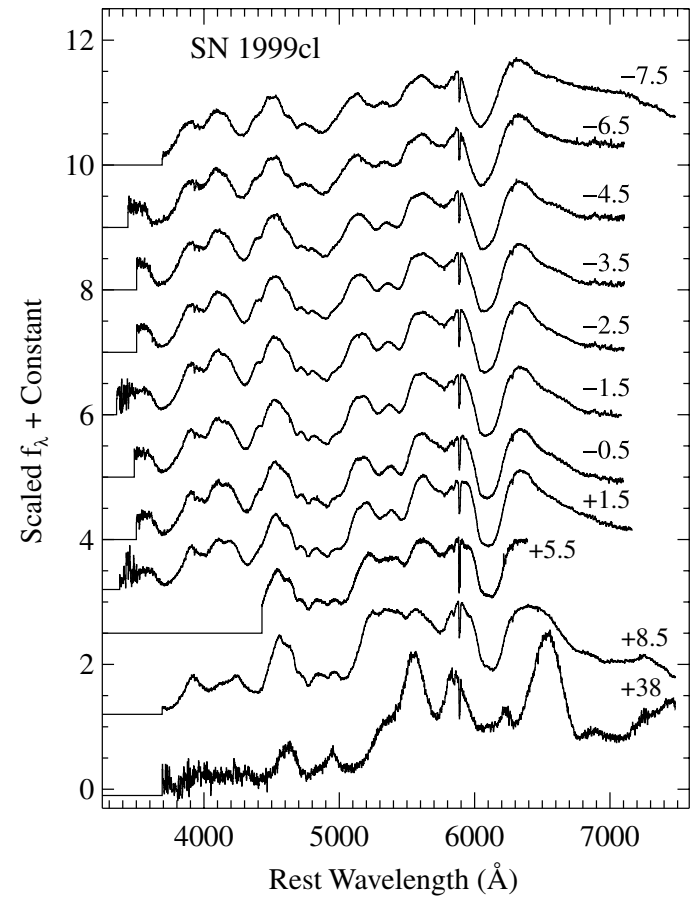

Figure 30. Spectra of SN 1999cl.

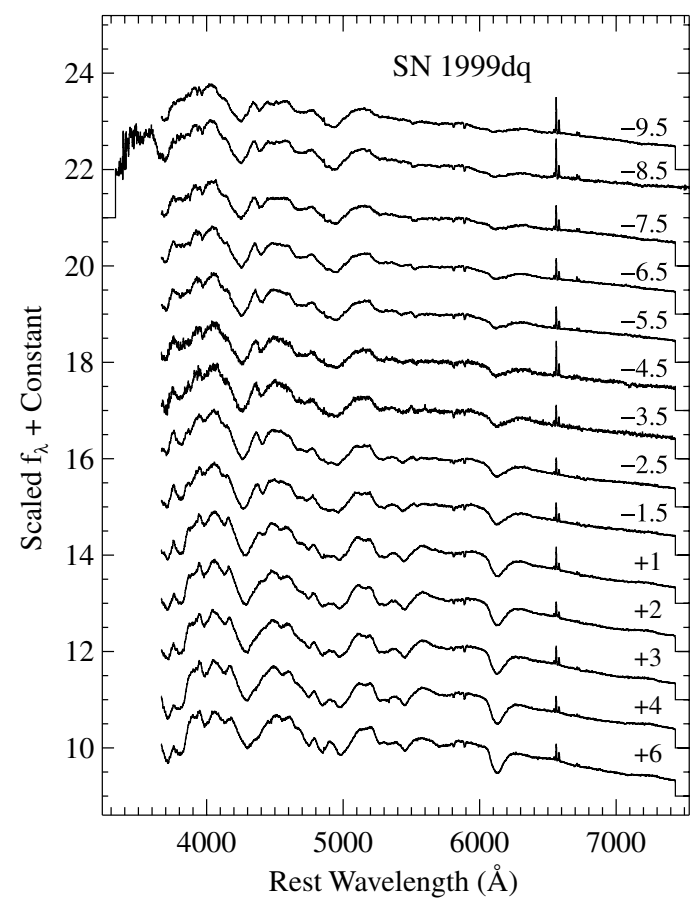

Figure 31. Early spectra of SN 1999dq.

\& Garnavich 1999). The Na I D line can be seen in the CfA spectra (Figure 34) and is at the host velocity. It has an EW of $4.7 \AA$. There are a limited number of spectra, the first at 3 days after maximum, with the rest mainly from a few weeks past maximum.

SN 1999gh. Nakano et al. (1999) reported the discovery of SN 1999gh on 1999 December 3. Subsequent spectroscopy showed that it was an SN Ia, and that the Si II $\lambda 5800$ line was stronger than usual, implying that this might be a subluminous SN Ia

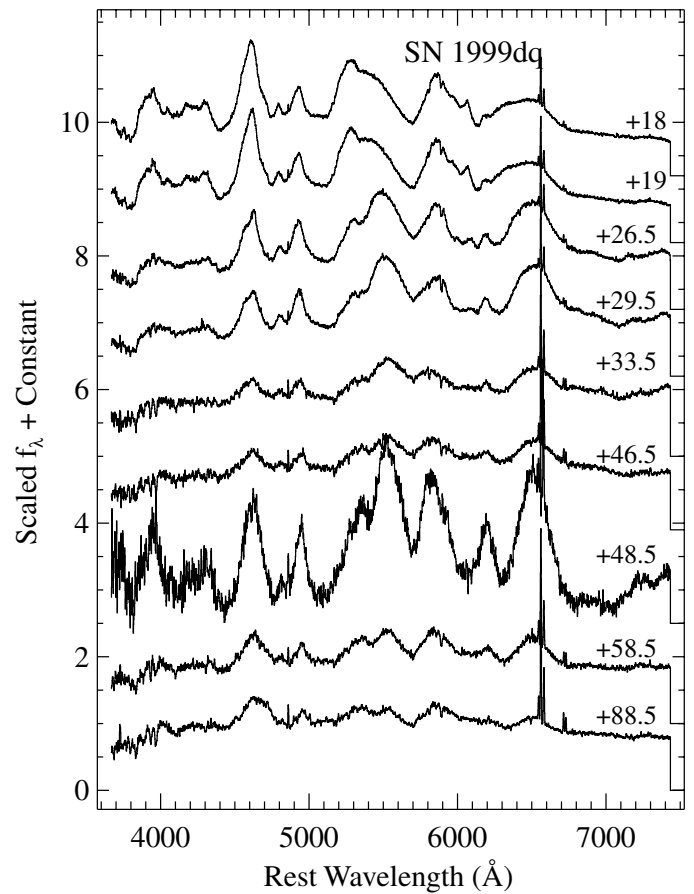

Figure 32. Late spectra of SN 1999dq. Note that for the days $+33.5,+46.5$, $+48.5,+58.5$, and +88.5 spectra, the relative strengths of the lines are dependent on galaxy contamination of the spectra. This can be affected by variable seeing and different observed position angles.

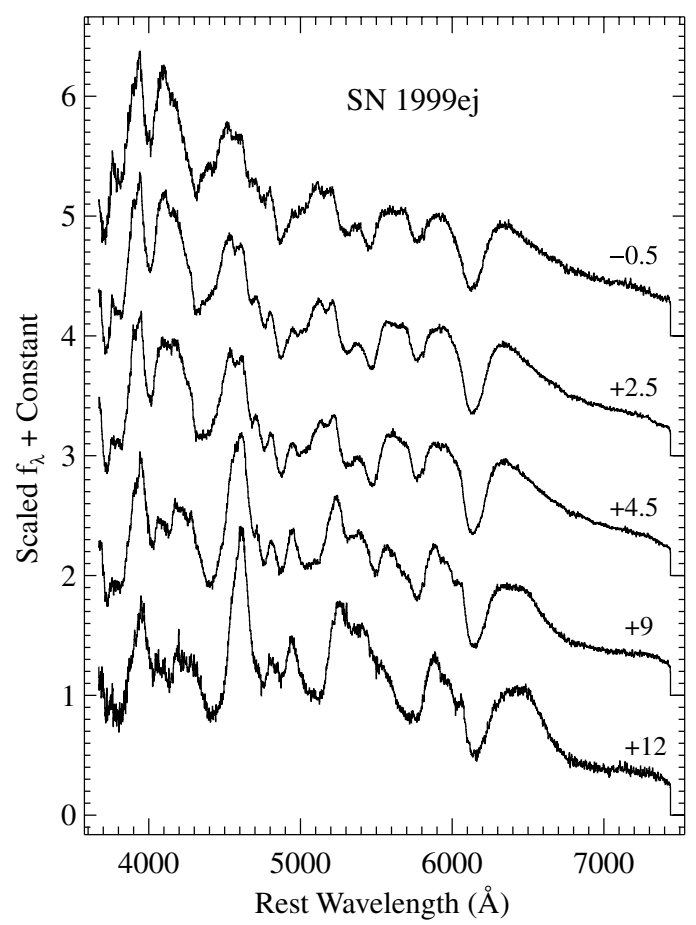

Figure 33. Spectra of SN 1999ej.

(Filippenko \& Garnavich 1999). We have an extensive set of spectra (Figure 35), but all from well past maximum (starting at 6 days after maximum). The $\Delta m_{15}(B)$ value of 1.69 (Jha et al. 2006) confirms that this was a peculiar SN Ia.

SN 1999gp. This SN was discovered by the LOSS on 1999 December 23 (Papenkova \& Li 1999). Our first CfA spectrum 


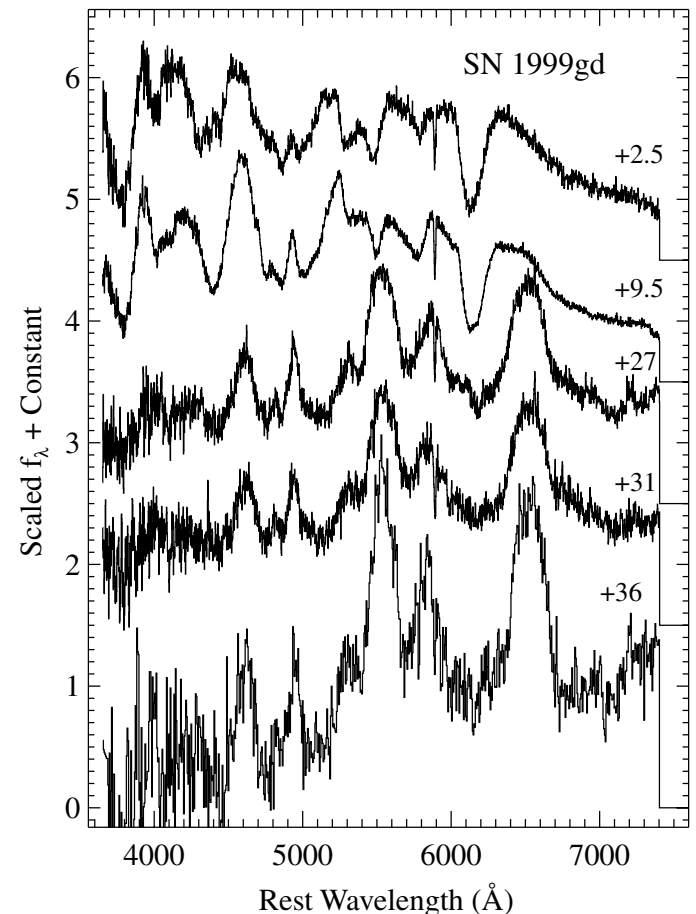

Figure 34. Spectra of SN 1999gd. The day +36 spectrum has been rebinned for clarity.

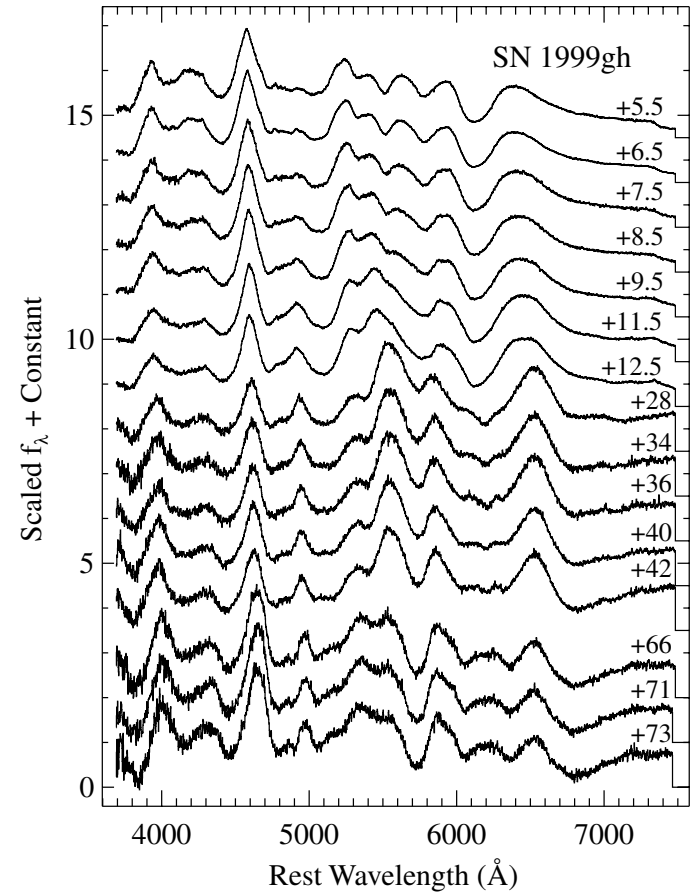

Figure 35. Spectra of SN 1999gh.

(Figure 36), obtained 5 days before maximum, was used to classify SN 1999gp as an SN Ia (Jha et al. 2000a). There is good coverage of the post-maximum decline as well as some spectra several weeks past maximum. Krisciunas et al. (2001) present optical and infra-red photometry of this SN.

SN 2000B. Antonini et al. (2000) found SN 2000B on 2000 January 13. Spectroscopy revealed that it was of Type Ia (Colas et al. 2000). The CfA spectra (Figure 37) are all from past

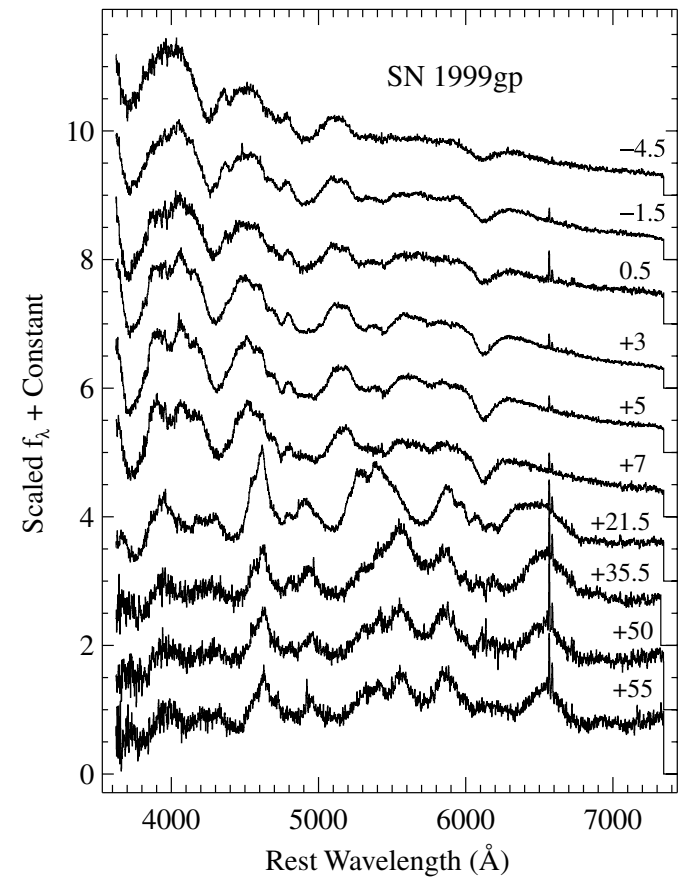

Figure 36. Spectra of SN 1999gp.

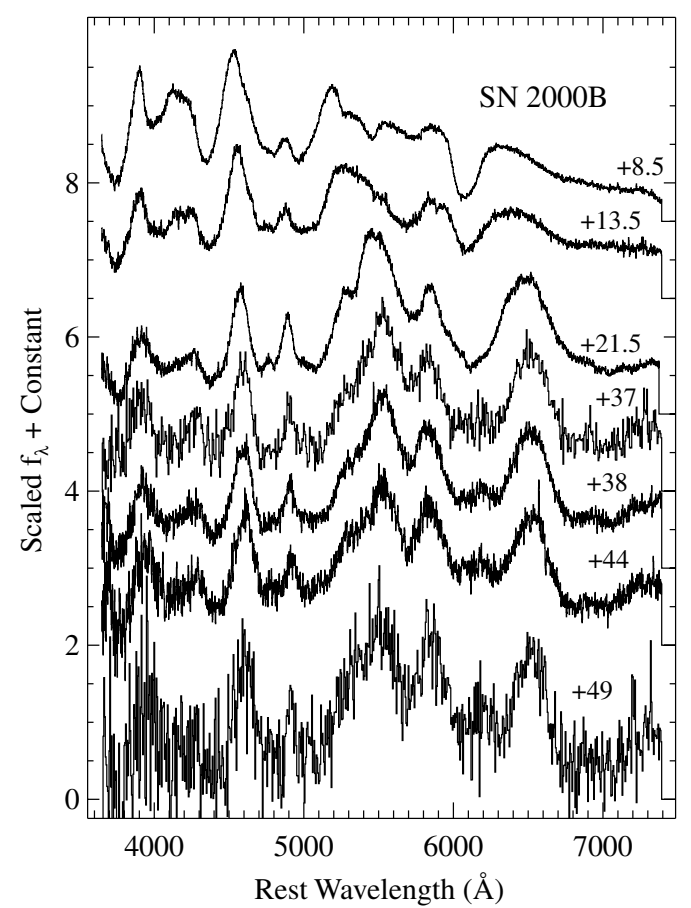

Figure 37. Spectra of SN 2000B. The days +37 and +49 spectra have been rebinned for clarity.

maximum, starting at 9 days after maximum, with most several weeks past maximum.

SN 2000cf. Puckett \& Sehgal (2000) discovered SN2000cf on 2000 May 9. Our first CfA spectrum (Figure 38), obtained 4 days after maximum, was used to classify SN 2000cf as an SN Ia (Jha et al. 2000c). There is limited spectroscopic coverage, all past maximum.

SN 2000cn. The LOSS found SN 2000cn on 2000 June 2 (Papenkova \& Li 2000). Two groups (including the CfA, using 


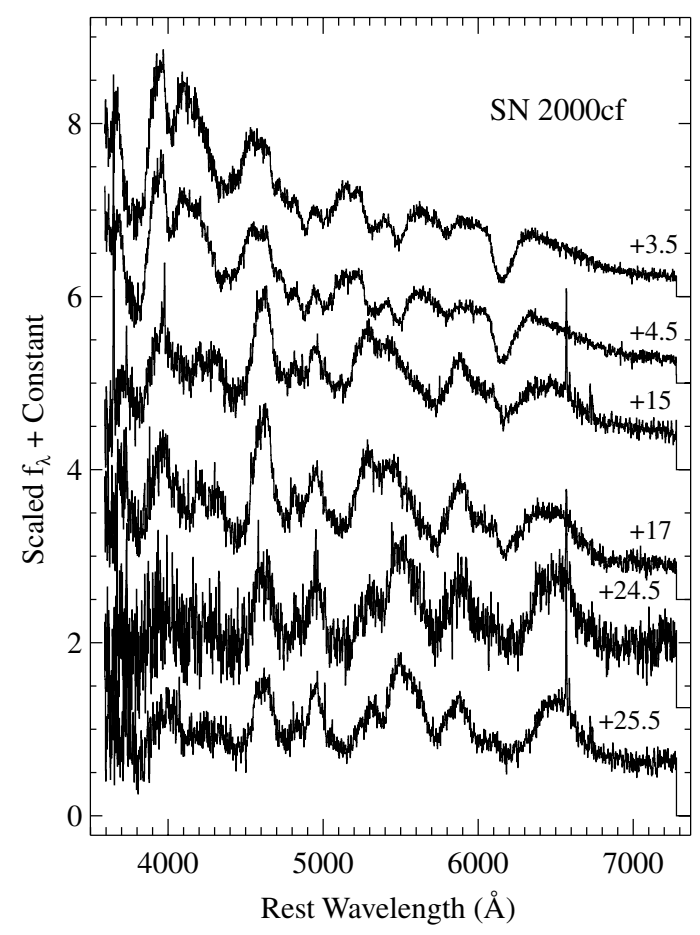

Figure 38. Spectra of SN 2000cf.

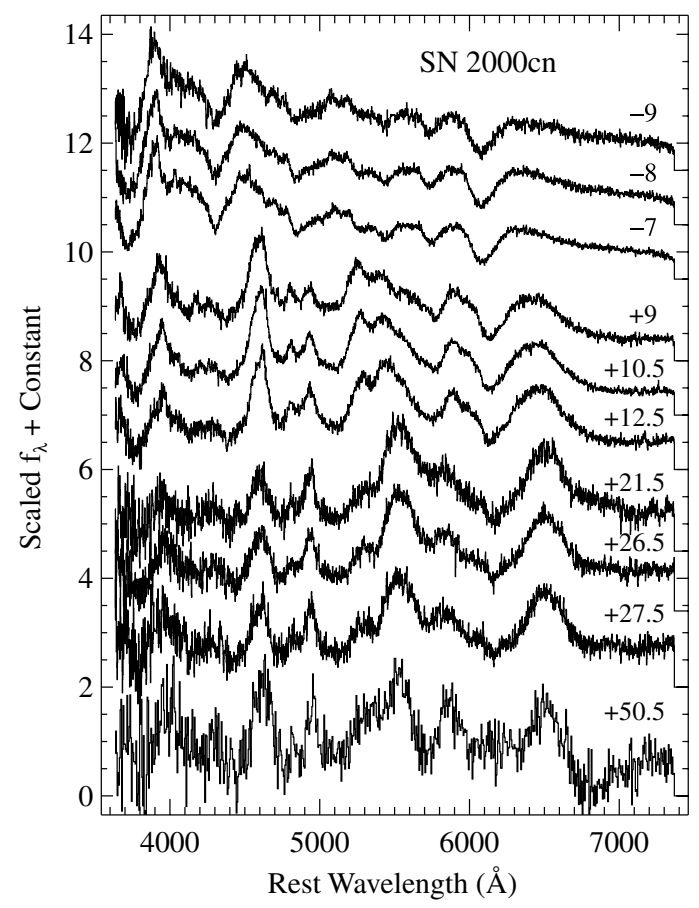

Figure 39. Spectra of SN $2000 \mathrm{cn}$.

our first spectrum obtained 9 days before maximum) reported that spectroscopy showed that this was an SN Ia (Jha et al. 2000b). Not noted in those initial reports, but apparent in our spectra (Figure 39), is the relative strength of Si II $\lambda 5800$ line. Photometry indicated $\Delta m_{15}(B)$ was 1.58 (Jha et al. 2006), confirming that this was a subluminous event. Many of the spectra are from several weeks past maximum, but there are a few at early epochs.

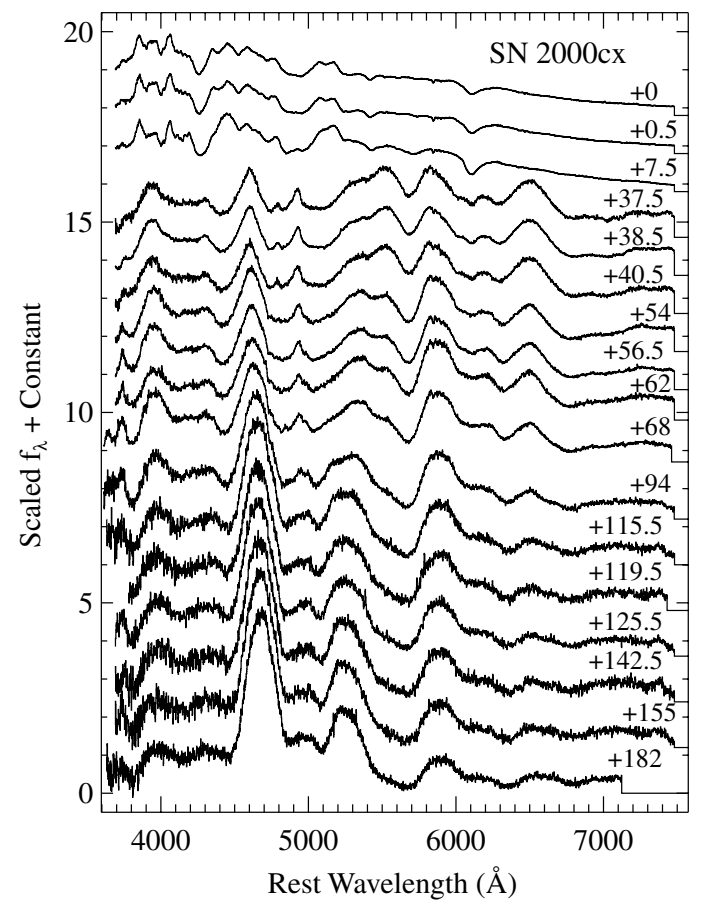

Figure 40. Spectra of SN 2000cx.

SN 2000cx. Another LOSS discovery, SN 2000cx was found on 2000 July 17 (Yu et al. 2000). Spectroscopy showed that it was an SN Ia, but with some peculiarities (Chornock et al. 2000). It resembled SN 1991T (Filippenko et al. 1992a; Phillips et al. 1992), with weak Si II $\lambda 6355$ and absorptions due to Fe III. This can be seen in the CfA spectra (Figure 40); there are a few near maximum, starting at maximum, but most are from several weeks to months past maximum, almost to the onset of the truly nebular phase (again as a result of the August shutdown at Mt. Hopkins). As discussed by Li et al. (2001a), SN 2000cx turned out to be so peculiar as to be unique. The light-curve shape did not match templates, and the spectroscopic evolution, while similar to SN 1991T, was also distinctive. This unusual SN elicited many further analyses (Cuadra et al. 2002; Rudy et al. 2002; Candia et al. 2003; Branch et al. 2004; Thomas et al. 2004; Sollerman et al. 2004).

SN 2000dk. The LOSS discovered SN 2000dk on 2000 September 18 (Beckmann \& Li 2000). Our first CfA spectrum (Figure 41), obtained 5 days before maximum, was used to classify SN 2000dk as an SN Ia (Jha et al. 2000d). In that report, it was not noted that the Si II $\lambda 5800$ line was relatively strong and that Ti II features were apparent, as can be seen in Figure 36. The $\Delta m_{15}(B)$ value of 1.57 (Jha et al. 2006) showed that this was a subluminous event. We have a few spectra around maximum, and a few at late times. Marion et al. (2003) show an infra-red spectrum of SN 2000dk.

SN 2000fa. Another LOSS discovery, SN 2000fa was found on 2000 November 30 (Friedman et al. 2000). Using our first CfA spectrum (Figure 42), this SN was classified as an SN Ia (Matheson et al. 2000b). We have many spectra from maximum through several weeks past maximum, as well as the first two, both obtained 10 days before maximum.

$S N 2001 \mathrm{~V}$. During the course of a redshift survey program at Mt. Hopkins, Jha et al. (2001) discovered SN 2001V spectroscopically on 2001 February 19. This spectrum revealed it to 


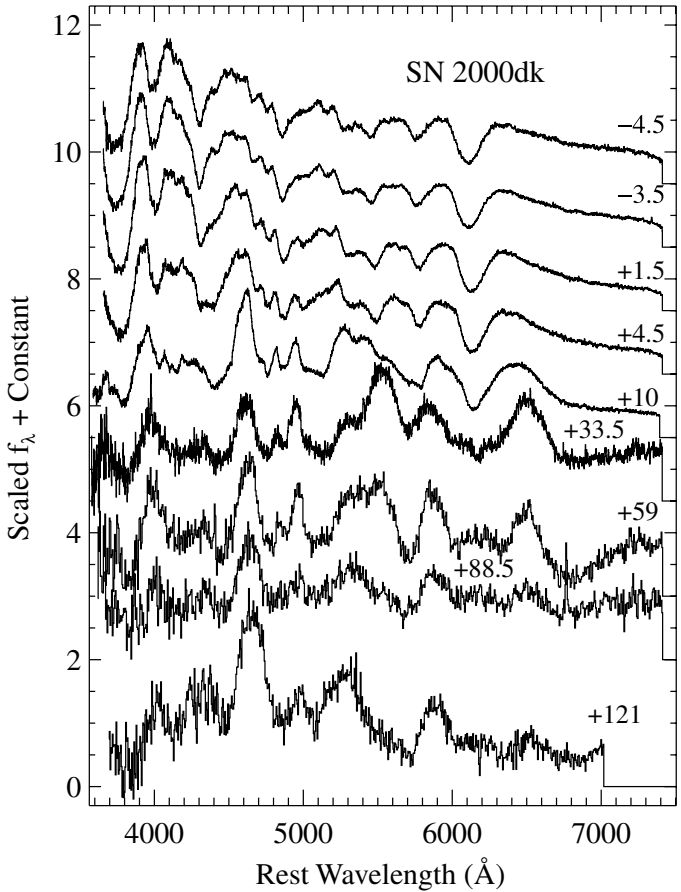

Figure 41. Spectra of SN 2000dk. The days $+59,+88.5$, and +121 spectra have been rebinned for clarity.

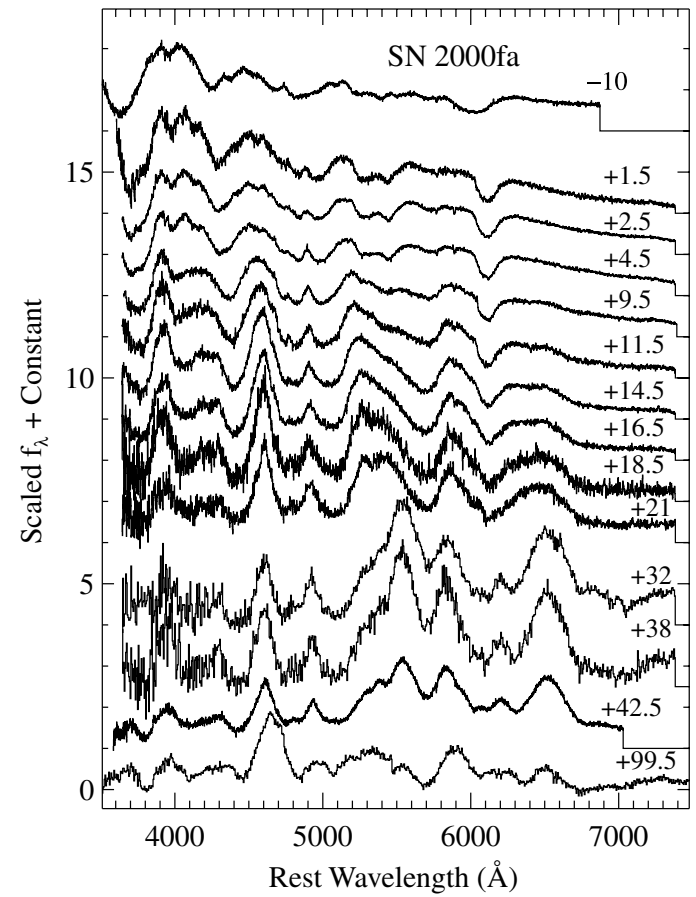

Figure 42. Spectra of SN 2000fa. The days $+32,+38$, and +99.5 spectra have been rebinned for clarity. In addition, the day +99.5 spectrum has been rescaled (so the zero point is not indicated).

be an SN Ia, at a very early epoch, 13 days before maximum. We were able to get extensive spectroscopic coverage of this $\mathrm{SN}$ (Figures 43 and 44) from 13 days before maximum to more than three months past maximum. Vinkó et al. (2003) also present photometry of SN $2001 \mathrm{~V}$.

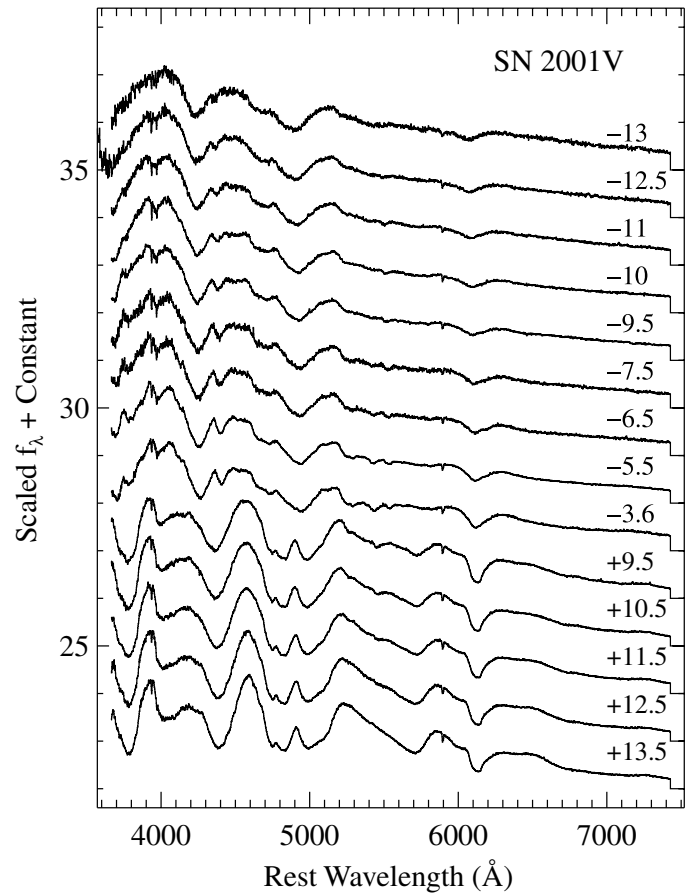

Figure 43. Early spectra of SN 2001V.

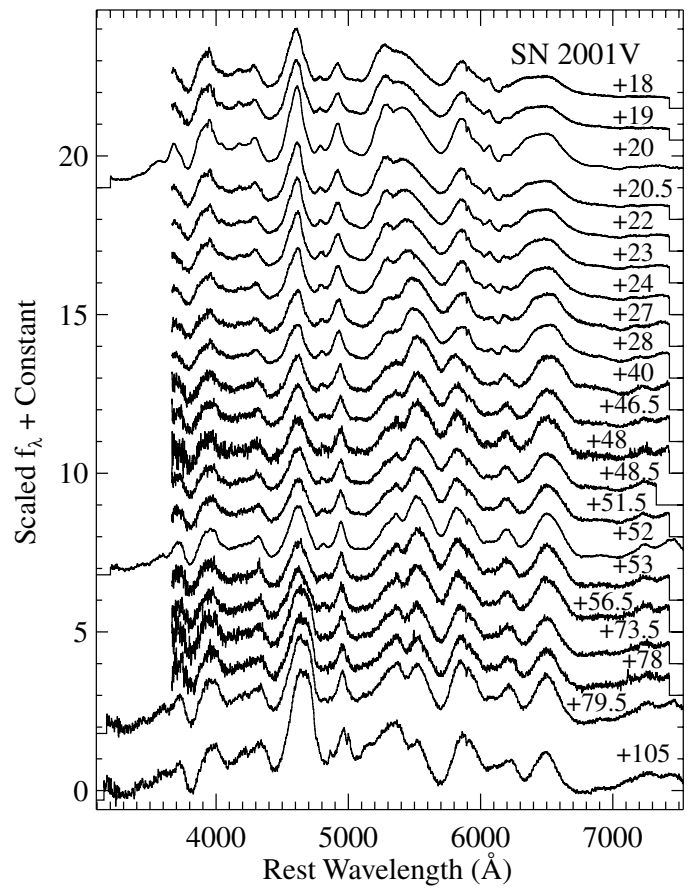

Figure 44. Late spectra of SN 2001V.

\section{CONCLUSIONS}

We have presented a large, homogeneous set of lowdispersion optical spectra of SNe Ia. All the SNe have wellcalibrated light curves with known properties such as the decline rate. The consistency of observation and reduction makes this an ideal sample for studying spectroscopic characteristics of SNe Ia in relation to the nature of their light curves (e.g., T. Matheson et al. 2008, in preparation). 
We would like to thank the staffs of the F. L. Whipple and MMT Observatories for their extensive assistance and support during this project. We would also like to thank Dan Koranyi and Barbara Carter for providing assistance with the observations. The CfA time allocation committee has been extremely generous in supporting SN observations at FLWO, especially in allowing the day-by-day monitoring of so many $\mathrm{SNe}$. This research was supported in part by the National Science Foundation under Grant No. PHY05-51164 to the KITP and AST-0606772 to Harvard University.

\section{REFERENCES}

Antonini, P., Colas, F., Frappa, E., \& Li, W. 2000, IAU Circ. 7347

Arbour, R., \& Armstrong, M. 1999, IAU Circ. 7108

Arbour, R., Papenkova, M., Li, W. D., Filippenko, A. V., \& Armstrong, M. 1999 IAU Circ. 7156

Astier, P., et al. 2006, A\&A, 447, 31

Ayani, K., et al. 1998, IAU Circ. 6905

Ayani, K., \& Yamaoka, H. 1998, IAU Circ. 6878

Beckmann, S., \& Li, W. D. 2000, IAU Circ. 7493

Benetti, S., et al. 2004, MNRAS, 348, 261

Benetti, S., et al. 2005, ApJ, 623, 1011

Bessell, M. S. 1999, PASP, 111, 1426

Branch, D. 2001, PASP, 113, 169

Branch, D., et al. 2003, AJ, 126, 1489

Branch, D., et al. 2004, ApJ, 606, 413

Branch, D., et al. 2005, PASP, 117, 545

Candia, P., et al. 2003, PASP, 115, 277

Chornock, R., Leonard, D. C., Filippenko, A. V., Li, W. D., Gates, E. L., \& Chloros, K. 2000, IAU Circ. 7463

Colas, F., Fienga, A., \& Buil, C. 2000, IAU Circ. 7351

Cuadra, J., Suntzeff, N. B., Candia, P., Krisciunas, K., \& Phillips, M. M. 2002, RevMexAA Conf. Ser., 14, 121

Fabricant, D., Cheimets, P., Caldwell, N., \& Geary, J. 1998, PASP, 110, 79

Falco, E. E., et al. 1999, PASP, 111, 438

Filippenko, A. V. 1982, PASP, 94, 715

Filippenko, A. V., \& De Breuck, C. 1998, IAU Circ. 6997

Filippenko, A. V., \& Garnavich, P. 1999, IAU Circ. 7328

Filippenko, A. V., Li, W. D., \& Leonard, D. C. 1999, IAU Circ. 7108

Filippenko, A. V., et al. 1992a, ApJ, 384, L15

Filippenko, A. V., et al. 1992b, AJ, 104, 1543

Friedman, A., King, J. Y., \& Li, W. D. 1999, IAU Circ. 7286

Friedman, A., Li, W. D., \& Schwartz, M. 2000, IAU Circ. 7533

Gamezo, V. N., Khokhlov, A. M., \& Oran, E. S. 2004, Phys. Rev. Lett., 92,211102

Garavini, G., et al. 2004, AJ, 128, 387

Garnavich, P., Jha, S., Kirshner, R., \& Berlind, P. 1998a, IAU Circ. 6980

Garnavich, P., Jha, S., Kirshner, R., \& Berlind, P. 1999a, IAU Circ. 7105

Garnavich, P., Jha, S., Kirshner, R., \& Berlind, P. 1999b, IAU Circ. 7159

Garnavich, P., Jha, S., Kirshner, R., Berlind, P., \& Calkins, M. 1998b, IAU Circ. 6858

Garnavich, P., Jha, S., Kirshner, R., Berlind, P., \& Calkins, M. 1998c, IAU Circ. 6880

Garnavich, P., Jha, S., Kirshner, R., \& Calkins, M. 1998d, IAU Circ. 6980

Garnavich, P., Jha, S., Kirshner, R., Challis, P., \& Berlind, P. 1999c, IAU Circ. 7169

Garnavich, P., Jha, S., Kirshner, R., Challis, P., \& Calkins, M. 1999d, IAU Circ. 7190

Garnavich, P. M., et al. 2004, ApJ, 613, 1120

Gerardy, C., \& Fesen, R. 1999, IAU Circ. 7158

Halderson, E., Modjaz, M., Shefler, T., King, J. Y., Papenkova, M., Li, W. D. Treffers, R. R., \& Filippenko, A. V. 1998, IAU Circ. 7050

Hamuy, M., Phillips, M. M., Suntzeff, N. B., Schommer, R. A., Maza, J., \& Aviles, R. 1996a, AJ, 112, 2391

Hamuy, M., et al. 1996b, AJ, 112, 2408

Hatano, K., Branch, D., Lentz, E. J., Baron, E., Filippenko, A. V., \& Garnavich, P. M. 2000, ApJ, 543, L49

Hillebrandt, W., \& Niemeyer, J. C. 2000, ARA\&A, 38, 191

Höflich, P., Gerardy, C. L., Fesen, R. A., \& Sakai, S. 2002, ApJ, 568, 791

Horne, K. 1986, PASP, 98, 609

Howell, D. A. 2001, ApJ, 554, L193

Howell, D. A., Höflich, P., Wang, L., \& Wheeler, J. C. 2001, ApJ, 556, 302
Hurst, G. M., \& Armstrong, M. 1998, IAU Circ. 6890

Hurst, G. M., Armstrong, M., \& Arbour, R. 1998a, IAU Circ. 6875

Hurst, G. M., Armstrong, M., Boles, T., Nakano, S., Kushida, Y., \& Kushida, R. 1998b, IAU Circ. 6841

Hurst, G. M., et al. 1998c, IAU Circ. 7033

Jha, S., Challis, P., Garnavich, P., Kirshner, R., \& Calkins, M. 1999a, IAU Circ. 7298

Jha, S., Challis, P., Kirshner, R., \& Berlind, P. 2000a, IAU Circ. 7341

Jha, S., Challis, P., Kirshner, R., Berlind, P., Turatto, M., Pastorello, A. Cappellaro, E., \& Cedrati, F. 2000b, IAU Circ. 7437

Jha, S., Challis, P., Kirshner, R., \& Calkins, M. 2000c, IAU Circ. 7423

Jha, S., Challis, P., Matheson, T., Kirshner, R., \& Berlind, P. 2000d, IAU Circ. 7494

Jha, S., Matheson, T., Challis, P., Kirshner, R., \& Berlind, P. 2001, IAU Circ. 7585

Jha, S., Garnavich, P., Challis, P., Kirshner, R., \& Berlind, P. 1998a, IAU Circ 7024

Jha, S., Garnavich, P., Challis, P., Kirshner, R., \& Berlind, P. 1999b, IAU Circ. 7250

Jha, S., Garnavich, P., Challis, P., Kirshner, R., \& Calkins, M. 1998b, IAU Circ. 6891

Jha, S., Garnavich, P., Challis, P., Kirshner, R., \& Calkins, M. 1998c, IAU Circ. 7054

Jha, S., Garnavich, P., Kirshner, R., Koranyi, D., \& Calkins, M. 1998d, IAU Circ. 6844

Jha, S., et al. 1999c, ApJS, 125, 73

Jha, S., et al. 2006, AJ, 131, 527

Jha, S., Riess, A. G., \& Kirshner, R. P. 2007, ApJ, 659, 122

King, J. Y., Shefler, T., Halderson, E., Li, W. D., Treffers, R. R., \& Filippenko, A. V. 1998, IAU Circ. 6991

Kirshner, R. P., \& Oke, J. B. 1975, ApJ, 200, 574

Kirshner, R. P., Oke, J. B., Penston, M. V., \& Searle, L. 1973, ApJ, 185, 303

Kirshner, R. P., et al. 1993, ApJ, 415, 589

Knop, R. A., et al. 2003, ApJ, 598, 102

Kotak, R., et al. 2005, A\&A, 436, 1021

Krisciunas, K., et al. 2001, AJ, 122, 1616

Krisciunas, K., et al. 2003, AJ, 125, 166

Kuchner, M. J., Kirshner, R. P., Pinto, P. A., \& Leibundgut, B. 1994, ApJ, 426, L89

Leibundgut, B., et al. 1993, AJ, 105, 301

Li, W., et al. 2001a, PASP, 113, 1178

Li, W., Filippenko, A. V., Treffers, R. R., Riess, A. G., Hu, J., \& Qiu, Y. 2001b, ApJ, 546, 734

Li, W. D. 1999a, IAU Circ. 7247

Li, W. D. 1999b, IAU Circ. 7319

Li, W. D., Modjaz, M., Halderson, E., Shefler, T., King, J. Y., Treffers, R. R., \& Filippenko, A. V. 1998, IAU Circ. 6978

Marion, G. H., Höflich, P., Vacca, W. D., \& Wheeler, J. C. 2003, ApJ, 591, 316

Massey, P., \& Gronwall, C. 1990, ApJ, 358, 344

Massey, P., et al. 1988, ApJ, 328, 315

Matheson, T., Filippenko, A. V., Ho, L. C., Barth, A. J., \& Leonard, D. C. 2000a, AJ, 120, 1499

Matheson, T., Jha, S., Challis, P., Kirshner, R., Huchra, J., \& Caldwell, N. 2000b IAU Circ. 7535

Mazzali, P. A., Cappellaro, E., Danziger, I. J., Turatto, M., \& Benetti, S. 1998, ApJ, 499, L49

Modjaz, M., Halderson, E., Shefler, T., King, J. Y., Li, W. D., Treffers, R. R., \& Filippenko, A. V. 1998a, IAU Circ. 6977

Modjaz, M., King, J. Y., Papenkova, M., Friedman, A., Johnson, R. A., Li, W. D., Treffers, R. R., \& Filippenko, A. V. 1999, IAU Circ. 7114

Modjaz, M., Li, W., Filippenko, A. V., King, J. Y., Leonard, D. C., Matheson, T., Treffers, R. R., \& Riess, A. G. 2001, PASP, 113, 308

Modjaz, M., Shefler, T., Halderson, E., King, J. Y., Li, W. D., Treffers, R. R., \& Filippenko, A. V. 1998b, IAU Circ. 6993

Nakano, S., \& Kushida, R. 1999, IAU Circ. 7109

Nakano, S., Takamizawa, K., Kushida, Y., \& Kushida, R. 1999, IAU Circ. 7328 Nomoto, K., Uenishi, T., Kobayashi, C., Umeda, H., Ohkubo, T., Hachisu, I., \& Kato, M. 2003, From Twilight to Highlight: The Physics of Supernovae (Berlin: Springer), 115

Oke, J. B. 1974, ApJS, 27, 21

Oke, J. B. 1990, AJ, 99, 1621

Oke, J. B., \& Gunn, J. E. 1983, ApJ, 266, 713

Papenkova, M., Filippenko, A. V., \& Treffers, R. R. 1999, IAU Circ. 7185

Papenkova, M., \& Li, W. D. 1999, IAU Circ. 7337

Papenkova, M., \& Li, W. D. 2000, IAU Circ. 7436

Patat, F., \& Maia, M. 1998, IAU Circ. 6890

Perlmutter, S., et al. 1997, ApJ, 483, 565 
Perlmutter, S., et al. 1999, ApJ, 517, 565

Phillips, M. M. 1993, ApJ, 413, L105

Phillips, M. M., Kunkel, W., \& Filippenko, A. V. 1999, IAU Circ. 7122

Phillips, M. M., Wells, L. A., Suntzeff, N. B., Hamuy, M., Leibundgut, B., Kirshner, R. P., \& Foltz, C. B. 1992, AJ, 103, 1632

Pignata, G., et al. 2004, MNRAS, 355, 178

Puckett, T., \& Sehgal, A. 2000, IAU Circ. 7421

Qiao, Q. Y., Qiu, Y. L., Li, W. D., Hu, J. Y., Esamdin, A., Wei, J. Y., Cao, L., \& Gu, Q. S. 1997, IAU Circ. 6775

Qiao, Q. Y., Wei, J. Y., Qiu, Y. L., \& Hu, J. Y. 1999, IAU Circ. 7109

Qiu, Y. L., Qiao, Q. Y., \& Hu, J. Y. 1998, IAU Circ. 7022

Qiu, Y. L., Qiao, Q. Y., Li, W. D., Hu, J. Y., Esamdin, A., \& Huang, K. L. 1997, IAU Circ. 6766

Riess, A. G., Press, W. H., \& Kirshner, R. P. 1996, ApJ, 473, 88

Riess, A. G., et al. 1998, AJ, 116, 1009

Riess, A. G., et al. 1999, AJ, 117, 707

Riess, A. G., et al. 2001, ApJ, 560, 49

Riess, A. G., et al. 2004, ApJ, 607, 665

Riess, A. G., et al. 2005, ApJ, 627, 579

Riess, A. G., et al. 2007, ApJ, 659, 98

Rudy, R. J., Lynch, D. K., Mazuk, S., Venturini, C. C., Puetter, R. C., \& Höflich, P. 2002, ApJ, 565, 413
Salvo, M., et al. 1998, IAU Circ. 7037

Schlegel, D. J., Finkbeiner, D. P., \& Davis, M. 1998, ApJ, 500, 525

Schmidt, G., Weymann, R., \& Foltz, C. 1989, PASP, 101, 713

Schwartz, M. 1999, IAU Circ. 7105

Sollerman, J., et al. 2004, A\&A, 428, 555

Stone, R. P. S. 1977, ApJ, 218, 767

Stritzinger, M., et al. 2002, AJ, 124, 2100

Stritzinger, M., Leibundgut, B., Walch, S., \& Contardo, G. 2006, A\&A, 450, 24 Suntzeff, N. B., et al. 1999, AJ, 117, 1175

Thomas, R. C., Branch, D., Baron, E., Nomoto, K., Li, W., \& Filippenko, A. V. 2004, ApJ, 601, 1019

Tonry, J. L., et al. 2003, ApJ, 594,

Toth, I., \& Szabó, R. 2000, A\&A, 361, 63

Villi, M. 1998, IAU Circ. 6899

Vinkó, J., Kiss, L. L., Csák, B., Fürész, G., Szabó, R., Thomson, J. R., \& Mochnacki, S. W. 2001, AJ, 121, 3127

Vinkó, J., et al. 2003, A\&A, 397, 115

Wade, R. A., \& Horne, K. D. 1988, ApJ, 324, 411

Wei, J.-Y., \& Li, W. D. 1998, IAU Circ. 6858

Wood-Vasey, W. M., et al. 2007, ApJ, 666, 694

Woosley, S., \& Weaver, T. A. 1986, ARA\&A, 24, 205

Yu, C., Modjaz, M., \& Li, W. D. 2000, IAU Circ. 7458 